ACCEPTED FOR PUBlication in ApJ

Preprint typeset using $\mathrm{AT}_{\mathrm{E} X}$ style emulateapj v. 11/10/09

\title{
STUDYING THE WARM-HOT INTERGALACTIC MEDIUM IN EMISSION
}

\author{
Y. TAkei ${ }^{1}$, E. URSino $^{2}$, E. Branchini ${ }^{2}$, T. Ohashi ${ }^{3}$, H. KaWAhara $^{3}$, K. Mitsuda $^{1}$,

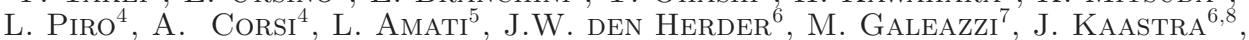 \\ L. Moscardini ${ }^{9,10}$, F. Nicastro ${ }^{11}$, F. Paerels ${ }^{12}$, M. Roncarelli ${ }^{9}$ and M. Viel ${ }^{13,14}$ ' \\ Accepted for publication in ApJ
}

\begin{abstract}
We assess the possibility to detect the warm-hot intergalactic medium (WHIM) in emission and to characterize its physical conditions and spatial distribution through spatially resolved X-ray spectroscopy, in the framework of the recently proposed DIOS, EDGE, Xenia, and ORIGIN missions, all of which are equipped with microcalorimeter-based detectors. For this purpose we analyze a large set of mock emission spectra, extracted from a cosmological hydrodynamical simulation. These mock X-ray spectra are searched for emission features showing both the O VII K $\alpha$ triplet and O VIII Ly $\alpha$ line, which constitute a typical signature of the warm hot gas. Our analysis shows that $1 \mathrm{Ms}$ long exposures and energy resolution of $2.5 \mathrm{eV}$ will allow us to detect about 400 such features per deg $^{2}$ with a significance $\geq 5 \sigma$ and reveals that these emission systems are typically associated with density $\sim 100$ above the mean. The temperature can be estimated from the line ratio with a precision of $\sim 20$ $\%$. The combined effect of contamination from other lines, variation in the level of the continuum, and degradation of the energy resolution reduces these estimates. Yet, with an energy resolution of $7 \mathrm{eV}$ and all these effects taken into account, one still expects about 160 detections per $\mathrm{deg}^{2}$. These line systems are sufficient to trace the spatial distribution of the line-emitting gas, which constitute an additional information, independent from line statistics, to constrain the poorly known cosmic chemical enrichment history and the stellar feedback processes.
\end{abstract}

Subject headings: cosmology: observations - intergalactic medium — large-scale structure of Universe - X-rays: diffuse background

\section{INTRODUCTION}

After more than 10 years, the so-called "missing baryon" problem is far from being solved. The original claim of Fukugita, Hogan, \& Peebles (1998) that the observational census of cosmic baryons in the local Universe falls short of the expected cosmologi-

takei@astro.isas.jaxa.jp

1 Institute of Space and Astronautical Science, Japan Aerospace Exploration Agency, 3-1-1 Yoshinodai, Chuo-ku, Sagamihara, Kanagawa 252-5210, Japan

2 Dipartimento di Fisica, Università degli Studi "Roma Tre" via della Vasca Navale 84, I-00146 Roma, Italy

3 Department of Physics, School of Science, Tokyo Metropolitan University, 1-1 Minami-Osawa, Hachioji, Tokyo 192-0397, Japan

${ }^{4}$ INAF-Istituto di Astrofisica Spaziale Fisica Cosmica, Via del Fosso del Cavaliere 100, I-00133 Roma, Italy

${ }^{5}$ INAF-Istituto di Astrofisica Spaziale e Fisica Cosmica Bologna, via P. Gobetti 101, I-40129 Bologna, Italy

6 SRON Netherlands Institute for Space Research, Sorbonnelaan 2, 3584 CA Utrecht, The Netherlands

7 Physics Department of University of Miami, 319 Knight Physics Building, Coral Gables, FL 33164, U.S.

8 Astronomical Institute, University of Utrecht, Postbus 8000 , 3508, TA Utrecht, The Netherlands

9 Dipartimento di Astronomia, Università di Bologna, via Ranzani 1, I-40127 Bologna, Italy

10 INFN/National Institute for Nuclear Physics, Sezione di Bologna, Via Berti Pichat 6/2, I-40127, Bologna, Italy

11 INAF-Osservatorio Astronomico di Roma,via Frascati 33, I00040 Monteporzio-Catone (RM), Italy

12 Columbia Astrophysics Laboratory and Department of Astronomy, Columbia University, 550 West 120th Street, New York, NY 10027, U.S.

13 INAF-Osservatorio Astronomico di Trieste, via Tiepolo 11, I-34131 Trieste, Italy

14 INFN/National Institute for Nuclear Physics, Via Valerio 2, I-34127 Trieste, Italy cal mean (Burles \& Tytler 1997; Barlow \& Tytler 1998; Spergel et al. 2003; Komatsu et al. 2009) has gained even more statistical significance (Fukugita \& Peebles 2004; Danforth \& Shull 2005) and it is now generally accepted that a large fraction of the baryons $(\sim 40 \%)$ is not accounted for by observations at $z \sim 0$. This fact is even more surprising if one considers the fact that the baryon density in the Ly- $\alpha$ forest at redshift $z=2-3$ (Weinberg et al. 1997; Rauch 1998) accounts for about all expected baryons whereas the nucleons in the Ly- $\alpha$ forest at $z \sim 0$ contributes to less than $30 \%$ of the cosmic mean (Bregman 2007).

Although baryons provide a small contribution to the cosmological mass-energy density, they are key players in forming stars and galaxies and determining the cosmochemical evolution of the diffuse gas. In addition, being the only component that interacts with the electromagnetic radiation, they are the sole probe to investigate the physics of the formation and evolution of visible cosmic structures and infer the co-evolution of the underlying dark matter distribution.

Cosmological numerical simulations have provided a solution to this apparent paradox suggesting that missing baryons at $z<1$ are distributed in a network of filaments with temperature $10^{5}-10^{7} \mathrm{~K}$ with density significantly above the cosmic mean but below that typically found in virialized structures like galaxy clusters. This phase is called warm-hot intergalactic medium (WHIM). These results, which constitute one of the rare examples in cosmology in which theory leads observations, explain why these baryons have escaped observations so far and indicate the best strategy to detect them. Indeed, the WHIM is expected to be so highly ionized that it can 
only be seen in the UV and X-ray bands but the signal should be very weak because of its relatively low density. Therefore it is not surprising that current observations in the UV and X-ray bands provide weak constraints to the WHIM models.

In the UV band, $\mathrm{O}$ VI absorption lines $(\lambda=$ 1032, $1038 \AA$ ) have been detected along the lines of sight to 50 AGN (e.g., Danforth \& Shull 2005, 2008; Tripp et al. 2008). Their cumulative number per unit redshift as a function of the line equivalent width (hereafter the $d N / d z$ statistics) are consistent with the predictions of numerical models (see e.g., Cen \& Fang 2006; Branchini et al. 2009). However, it is debated how reliably the observed O VI absorption lines trace the WHIM since their width seems too small to be produced by warm-hot material. This means that either the observed lines trace the local Ly- $\alpha$ forest rather than the WHIM or that the gas is two-phased: a photo-ionized gas responsible for the Ly- $\alpha$ absorption and shock-heated WHIM responsible for the O VI lines. Recent numerical simulations carried out to investigate the physical properties of the IGM have helped to clarify the issue (????). These simulations have shown that the thermal state of the IGM at $z \sim 0$ is significantly affected by a number of physical processes like galactic wind, AGN feedback, metal line cooling, photoionization by background radiation, sub-resolution turbulence. Although it is non trivial to compare the results of different works and not all these works seem to converge to the same results, it is remarkable that two of them (??) indicate that about a third of the OVI absorbers are photo-ionized and trace the Ly- $\alpha$ forest at $z \sim 0$ while the rest of them trace the WHIM. An upper limit can nevertheless been worked out: if all gas is multi-phased, i.e., in the limit in which all the observed O VI lines are produced by the WHIM, then the O VI can probe no more than $7-10 \%$ of the baryons (Danforth \& Shull 2005; Tripp et al. 2006; Danforth 2009). With the inclusion of the thermally broadened Ly $\alpha$ absorbers (BLAs) recently observed by Danforth et al. (2010) and Naravanan et al. (2010a,b), these estimates are boosted up by a factor of $\sim 2$, i.e., UV observations are capable of probing $\sim 20 \%$ of the baryons in the local universe (Danforth et al. 2010). Nevertheless, whereas O VI might be a useful signpost for a non negligible fraction of the missing baryons, the bulk of the WHIM cannot be investigated through UV spectroscopy. $\mathrm{X}$-ray band observations are required.

In the soft X-ray band, the residual unresolved background after point-sources subtraction possibly constitutes the second observational evidence of the WHIM. So far the tightest constraints have been obtained by Hickox \& Markevitch (2007a,b) after removing all contributions associated to X-ray, optical, and IR sources in the Chandra Deep Fields. Their analysis has shown that the residual background in the $0.65-1 \mathrm{keV}$ energy band cannot be accounted for by AGN below the resolution limit. Instead, it appears to be consistent with the expected integrated emission contributed by the WHIM, as predicted by models based on hydrodynamical simulations (e.g., Roncarelli et al. 2006). The intensity of the diffuse X-ray background constitutes a weak constraint on the WHIM properties. Additional constraints can be obtained by identifying the WHIM signature in the an- gular correlation properties of the X-ray background, like in the recent analysis of Galeazzi et al. (2009).

Hydrodynamical simulations show that the best way to investigate the WHIM is by observing the X-ray emission or absorption lines of highly ionized element like $\mathrm{C}, \mathrm{N}, \mathrm{O}, \mathrm{Ne}, \mathrm{Mg}$, and Fe. Deep X-ray spectroscopy has indeed been performed to detect such lines. ¿From a theoretical viewpoint the possibility of detecting and studying the WHIM in the absorption spectra of Xray bright objects like AGN or Gamma-ray bursts has been thoroughly discussed both from a general perspective (e.g., Kravtsov et al. 2002; Klvpin et al. 2003; Chen et al. [2003; Viel et al. 2005; Cen \& Fang 2006) and by considering observations carried out with some specific instrument (Viel et al. 2003; Yoshikawa et al. 2003, 2004; Kawahara et al. 2006; Paerels et al. 2008; Branchini et al. 2009). ¿From the observational side, the analysis of the absorption spectra has lead to several claimed WHIM detections (e.g., Fang et al. 2002; Mathur et al. 2003; Nicastro et al. 2005) whose statistical significance has been questioned by subsequent studies (Rasmussen et al. 2007; Kaastra et al. 2006), reflecting the fact that these measurements are at the limits of current instrumental capabilities. The attempts to detect absorption lines associated to dense parts of large-scale structure leads to marginal detections with less than $4 \sigma$ significance (Fuijimoto et al. 2004; Takei et al. 2007; Buote et al. 2009; Fang et al. 2010; Zappacosta et al. 2010).

Studying the WHIM in absorption offers advantages and disadvantages. The advantage is that line detection depends on the source flux with no need to worry about foreground contributions. In addition, the line strength depends on the density of the intervening absorber, allowing one to probe regions of moderate overdensity. The obvious drawback is that one can only probe the WHIM along the few lines of sight to X-ray bright objects, making it very difficult to trace its spatial distribution (but see Viel et al. 2003, for a possible way of probing the WHIM clustering in absorption).

On the other hand, studying the WHIM in emission is considerably harder but potentially more rewarding. The main difficulty is represented by the fact that line detection depends now on the capability of modeling the various contributions to the soft Xray foreground, which, unfortunately, are poorly constrained by observational data (McCammon et al. 2002; Smith et al. 2005; Henlev et al. 2007; Smith et al.|2007; Henley \& Shelton 2008; Gupta et al. 2009; Yoshino et al. 2009). It is therefore not surprising if only a few WHIM detections have been claimed in continuum emission (Zappacosta et al. 2005; Mannucci et al. 2007; Takei et al. 2007; Werner et al. 2008). The main reward is constituted by the possibility of tracing the 3D distribution of the WHIM (Cen \& Fang 2006) and quantifying its spatial and angular correlation properties (Ursino \& Galeazzi 2006; Ursino et al. 2010b). In addition, since X-ray emissivity scales with the square of the gas density, we expect the emission signal to be dominated by high density regions, effectively complementing the absorption studies.

In this work we will investigate the possibility of detecting, studying and characterizing the WHIM in emission. In order to observe the weak WHIM emission features a 
detector with a large grasp (effective area $\times$ field of view) is mandatory. In addition, to separate the WHIM lines from other spectral features contributed by other sources a good energy resolution is also required. A number of recently-proposed X-ray satellite missions such as DIOS (Ohashi et al. 2010), EDGE (Piro et al. 2009), Xenia (Burrows et al. 2010), and ORIGIN meet these requirements. We will assume observations with the detectors on board the proposed EDGE and Xenia missions. The detailed characteristics of these two X-ray satellites are presented in Piro et al. (2009)15, and in Burrows et al. (2010). For our purposes, the most relevant instrument is the cryogenic microcalorimeter-based spectrometer array: CRIS (Cryogenic Imaging Spectrometer). CRIS has the energy range of $0.2-2.2 \mathrm{keV}$ (goal $0.1-3 \mathrm{keV}$ ), the energy resolution of $\Delta E=2.5 \mathrm{eV}$ (goal $1 \mathrm{eV}$ ) at $5.9 \mathrm{keV}$, the FOV of $0.9^{\circ} \times 0.9^{\circ}$ (goal $1.1^{\circ} \times 1.1^{\circ}$ ), the effective area $A=1000 \mathrm{~cm}^{2}$ (goal $1300 \mathrm{~cm}^{2}$ ) at $0.5 \mathrm{keV}$, and the angular resolution (HPD) of $\Delta \theta=4^{\prime}$ (goal 2.5'). DIOS spectrometer has a similar energy resolution but a factor of 10 smaller grasp than CRIS onboard EDGE or Xenia. $O R I G I N$ is planned to have a better angular resolution $<30^{\prime \prime}$, at the expense of energy resolution $(\Delta E=3-5$ $\mathrm{eV})$. In this work we refer to the adopted baseline of the EDGE and Xenia spectrometer as a reference case and explore the effect of a different energy resolution, chosen in the range [1-7] eV. In contrast, we do not explore the angular resolution better than $1.3^{\prime}$, because the typical angular size of the WHIM above detection threshold is $>2^{\prime}-3^{\prime}$.

The layout of this paper is as follows. In $\S 2$ we introduce the WHIM model used in this work from which we obtain the simulated surface brightness maps and emission spectra analyzed in this work. In $\S 3$ the problem of detecting the WHIM emission lines and computing their relevant statistics is addressed. In $\S 4$ we study the physical properties of the regions in which the WHIM can be detected in emission and assess the possibility of measuring the gas temperature from line ratios. In $\S 5$ we investigate the possibility of tracing the spatial distribution of the WHIM. In $\S[$ ] we quantify the impact of energy resolution and line contamination form different sources. In $\S$ 7, we address the question of to what extent we can discriminate among different models of stellar feedback and metal diffusion, using emission studies. Finally we discuss the main results and conclude in $\S 8$.

\section{MODELING THE WHIM}

Hydrodynamical simulations provide the best way of studying the properties of the WHIM (see, e.g., Cen \& Ostriker 1999a,b; Davé et al. 2001; Chen et al. 2003; Borgani et al. 2004; Cen \& Ostriker 2006: Oppenheimer \& Davé 2008; Bertone et al. 2010; Tornatore et al. 2010). Their analysis has revealed that the missing baryon problem finds its natural solution in a standard $\Lambda$ CDM framework in which the WHIM is heated up to temperature of $10^{5}-10^{7} \mathrm{~K}$ mostly by means of hydrodynamical shocks resulting from the build-up of cosmic structures at scales that are entering the nonlinear regime of density fluctuations growth. Additional heating/cooling mechanisms like stellar and AGN feedback, galactic superwinds, and radiative cooling,

\footnotetext{
15 Also see http://projects.iasf-roma.inaf.it/EDGE
}

and ionization by background radiation further modify the thermal state of the gas. Despite the recent advances in modeling these mechanisms Cen \& Ostriker 2006; Cen \& Fang 2006; Bertone et al. 2010; Tornatore et al. 2010; Shen et al. 2010; ?; ?; ?; ?) we still do not have a firm physical understanding of the effects of stellar feedback, chief among which is metal enrichment.

Indeed, the metal enrichment is so important in determining the observational properties of the WHIM that, following Branchini et al. (2009), we prefer to specify gas metallicity after the simulation run (i.e in the postprocessing phase) rather than self-consistently during the numerical simulation. The resulting WHIM model has been shown to fulfill all existing observational constraints ranging from the $d N / d z$ of the O VI and O VII absorption lines to the surface brightness of the diffuse X-ray background. So, although effective, the WHIM model used in this work is not self-consistent. However, given the existing uncertainties in predicting metal abundances, in this work we will not rely on a single, sophisticated WHIM model. Instead, in an attempt to account for theoretical uncertainties, we will consider two WHIM models that have been obtained from the same hydrodynamical simulations but adopting different prescriptions for the gas metallicity.

Our WHIM models have been obtained from the hydrodynamical simulation of Borgani et al. (2004) performed with the GADGET-2 Lagrangian code (Springel 2005) in a computational box of size $192 h^{-1}$ comoving Mpc, loaded with $480^{3}$ Dark Matter and $480^{3}$ gas particles. Dark matter and gas particles have masses of $4.62 \times 10^{9} h^{-1} M_{\odot}$ and $6.93 \times 10^{8} h^{-1} M_{\odot}$, respectively. The Plummer-equivalent gravitational softening is $\epsilon=7.5 h^{-1} \mathrm{kpc}$ at $z=0$, fixed in physical units between $z=2$ and $z=0$, i.e., the redshift interval relevant for the study of the WHIM. The background cosmological model is a flat $\Lambda$ CDM with $\Omega_{\Lambda}=0.7, \Omega_{\mathrm{b}}=0.04$, $H_{0}=70 \mathrm{~km} \mathrm{~s}^{-1} \mathrm{Mpc}^{-1}(h=0.7)$, and power spectrum normalization $\sigma_{8}=0.8$. This numerical experiment uses simple recipes to account for the main non-gravitational heating and cooling mechanisms, namely: i) the star formation process that is treated by adopting a subresolution multiphase model for the interstellar medium (Springel \& Hernquist 2003), ii) the feedback from SNe that includes the effect of weak galactic outflows and iii) the radiative cooling assuming zero metallicity and heating/cooling from the photo-ionising UV background by Haardt \& Madau (1996). Finally, to construct our mock emission spectra we have considered all available simulation outputs out to $z=0.5$.

As mentioned above, the gas metallicity has been specified in the post-processing phase and, to account for the scatter in model predictions, we have explored two different scenarios. In the first one, which we call model B1, we have adopted the deterministic metallicity-density relation $Z=\min \left(0.3,0.005\left(1+\delta_{q}\right)^{1 / 2}\right)$, where $\delta_{g}$ is $\rho_{\text {gas }} /\left\langle\rho_{\text {gas }}\right\rangle-1$, that Croft et al. (2001) have proposed to match the average relation measured in numerical experiments. This is a conservative model that typically underestimates the line emissivity since it ignores the large scatter in the $Z-\delta_{g}$ relation which would enhance the contribution of the line emission from regions characterized by moderate gas overdensity, like the WHIM. For 
this reason, the B1 model should be regarded as a generous lower bound to our detectability estimates. In the second model, dubbed B2, we do account for the large scatter in the metallicity-density relation. This scatter reflects the fact that the metal abundance at a given location is not simply determined by the underlying gas density but also by the thermal and chemical history of the gas particles. Since it would be hard to model this scatter analytically we resort to hydrodynamical simulations. For this reason we have enforced the same $Z-\delta_{g}$ relation measured by Cen \& Ostriker (1999a b) in their numerical experiment. In practice we use the density-metallicity scatter plot measured at $z=0$ in the Cen \& Ostriker (1999b) simulation to derive a 2D probability distribution which is then fed into a Monte Carlo procedure to assign metallicity to gas particles with known density in the Borgani et al. (2004) simulation. The result is a metallicity model with the same $Z-\delta_{g}$ relation plotted in Fig. 2 of Cen \& Ostriker (1999a) and characterized by a $1 \sigma$ scatter of $\sim 0.3$ dex. Note that we did not use the metallicity of the Borgani et al. (2004) simulation itself since the metal diffusion mechanism built-in the SPH code reproduces the correct metal content in high density regions, like the intracluster medium, but systematically underestimates the metal abundance in the typical WHIM environments.

We stress the fact that the metallicity assigned in the post-processing is not self-consistent with the thermal state of the gas which, in the simulation considered in this work, is determined ignoring the role of metal cooling. In fact, recent numerical experiments show that the thermal state of the diffuse gas can be significantly altered by metal cooling (????). However, including the effect of metal cooling is beyond the scope of this paper. Instead, we attempt to bracket the uncertainties that derives from our simplified by adopting the two very different density-metallicity relations, being confident that the deterministic density-metallicity relation will provide very conservative predictions for the detectability of the WHIM.

To determine the ionization balance we have assumed pure collisional ionization equilibrium (CIE) and not hybrid collisional and photoionization equilibrium as in Branchini et al. (2009). The use of the CIE assumption is justified by the fact that in this work we are interested in emission spectra whose intensity scales with the squared density of the gas and thus it is heavily weighted towards high density peaks where the ionisation is basically determined by collisions. Branchini et al. (2009) were interested in absorption lines which typically trace the WHIM in regions of moderate overdensity where photo-ionization cannot be neglected. Here we focus on WHIM lines For this reason we have assumed CIE and used the APEC thermal model included in the code XSPEC (Smith et al. 2001) to compute emission spectra (Ursino \& Galeazzi 2006). The resulting mock Xray spectra contains all metal lines but here we focus on the strongest ones only: the O VIII Ly $\alpha$ line $(E=0.653$ $\mathrm{keV}$ ) and the $\mathrm{O}$ VII $\mathrm{K} \alpha$ triplet $(\mathrm{E}=0.561,0.569$, and $0.574 \mathrm{keV})$.

Model B2 agrees with all available observational constraints and, from a theoretical viewpoint, predicts a phase-space diagram (shown in Figure 6 of Branchini et al. (2009)) which is remarkably close to that

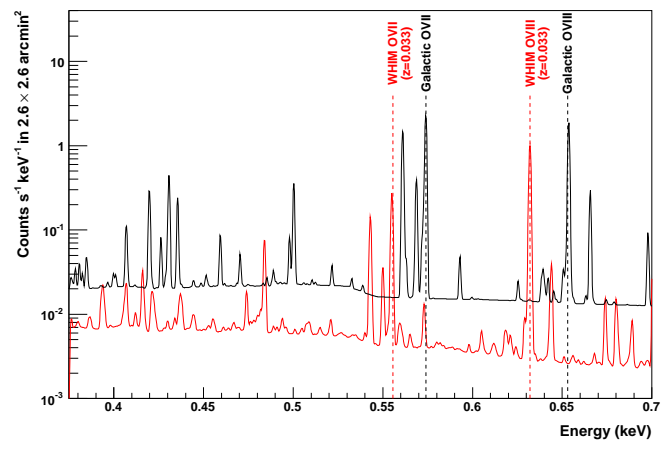

FIG. 1. - Emission spectrum in a $2.6^{\prime} \times 2.6^{\prime}$ area taken in a 1 Ms observation with Xenia CRIS, assuming an energy resolution $\Delta E=1 \mathrm{eV}$. Black: Sum of the Galactic Foreground and unresolved extragalactic background. The Galactic O VII triplet and the O VIII K $\alpha$ line at $z=0$ are flagged. Red: Contribution from the extragalactic gas. The O VII and O VIII lines at $z=0.033$ are indicated in the plot.

of Cen \& Ostriker (2006) (shown in Figure 6 of that paper), a fact that further increases our confidence on model B2. Therefore in this work we consider B2 as the reference model and regard predictions from model B1 as a generous lower bound.

One final caveat for model B2: this model is known to overestimate the X-ray emissivity in high $\left(\delta_{g}>10^{3}\right)$ density environments, i.e. emission from gas in groups and clusters (Ursino et al. 2010a). While the precise reason for this systematics is unknown, a plausible explanation is represented by the fact that in our model we have assumed primordial composition in the cooling function, instead of updating the cooling rate according to the evolving metallicity of the gas. The analysis of Bertone et al. (2010) suggests that this would artificially increase the emissivity in regions with high density. Brighter emission from group-like environments increases the chance superposition from strong emission lines, like those of the $\mathrm{Fe}$ L complex, that may contaminate or even outshine the weaker $\mathrm{O}$ lines, artificially reducing our theoretical estimate of WHIM detection. We will quantify these effects in $\S 6.1$ We again stress the fact that the inclusion of metal line cooling might have an impact on both the line surface brightness and the thermal state of the gas. We qualitatively discuss the expected impact of metal cooling in $\oint 8$.

\subsection{Mock emission spectra}

The detailed procedure used to produce the mock spectra is described in detail in Ursino et al. (2010b), here we provide a brief summary. To compute the mock Xray spectra we have considered all gas particles within a light-cone extracted from the hydrodynamical simulation, out to $z=0.51$. The light cone is formed by stacking the simulation outputs corresponding to 7 redshift intervals, each one of them corresponding to a comoving depth of $192 h^{-1} \mathrm{Mpc}$. We do not consider the WHIM at $z>0.5$ since in that case the O VII lines would be redshifted to energies at which a number of emission lines due to $\mathrm{L}$ and $\mathrm{M}$ transitions of heavier metals make identification of $\mathrm{O}$ lines difficult. In the stacking process, the simulation cubes that constitutes the individual redshift intervals were randomly shifted and rotated to avoid periodic replicas of the same large scale structures. Then, after selecting a random observer within the cube 
at $z=0$ we generate a single cone of view of $5.5^{\circ} \times 5.5^{\circ}$ and tag gas particles with their redshift, angular position, density, and temperature. Particles with temperature in the range $10^{5}-10^{7} \mathrm{~K}$ and overdensity $\delta<1000$ are classified as "WHIM phase". Among the remaining gas particles, those that provide a significant contribution to line emission are either in a "hot phase" $(\delta \geq 1000$ and $T \geq 10^{7} \mathrm{~K}$, roughly corresponding to gas within clusters of galaxies $)$ or in a "dense phase" $\left(\delta \geq 1000, T<10^{7}\right.$ $\mathrm{K}$, typically associated to galaxy groups). Their contribution to the X-ray emission is included in our mock spectra. The contribution from particles colder than $10^{5}$ $\mathrm{K}$ is negligible. In the mock spectra we did not include the contribution from gas at $z>0.51$. Emission lines (like the $\mathrm{Fe} \mathrm{L}$ complex in galaxy groups) from high- $z$ gas potentially contaminates spectra in the energy range in which we search for WHIM lines. We estimate the impact of contamination by spurious lines in $\S 6.1$.

To account for the finite angular resolution of the instrument, the FOV of the light cone is divided into $256 \times 256$ pixels of $1.3^{\prime} \times 1.3^{\prime}$, i.e. smaller than the planned CRIS resolution but possibly consistent with alternative instrument designs. The mock X-ray spectrum within each angular resolution element was obtained by adding together the individual spectra from each gas particle within the pixel plus that of gas particles in the contiguous pixels, weighted according to the smoothing kernel. The mock spectra were then sampled with an energy resolution of $1 \mathrm{eV}$ to mimic the energy resolution of the instrument. The effect of the Galactic absorption has been included using the model of Morrison \& McCammon (1983) in which we have adopted typical high latitude column density value of $1.8 \times 10^{20} \mathrm{~cm}^{-2}$ (McCammon et al. 2002). We have assumed the same metal solar abundance as in the Anders \& Grevesse (1989) models. The final result is a suite of $256 \times 256 \times 7$ mock spectra that we search for detectable WHIM lines. A typical example of mock spectrum is shown in Fig. 11 The red curve represents the contribution by extragalactic gas in a light cone with FOV of $2.6^{\prime} \times 2.6^{\prime}$ (i.e. the sum of $2 \times 2 \times 7$ individual mock spectra). Photon counts have been computed in $\Delta E=1 \mathrm{eV}$ energy bins assuming a $1 \mathrm{Ms}$ observation with CRIS. The spectrum is convolved with the detector response matrix of $1 \mathrm{eV}$ energy resolution (FWHM). Emission lines from different metals are clearly visible. In particular we have flagged those lines corresponding to a line system at $z=0.033$. The O VII triplet and the O VIII recombination line are clearly visible. The black curve shows the sum of the contributions of the Galactic foreground and the unresolved extragalactic background modeled after McCammon et al. (2002). The Galactic $\mathrm{O}$ VII and O VIII lines at $z=0$ are clearly seen in correspondence of their rest frame energy.

\section{ANALYSIS OF THE MOCK SPECTRA}

To assess the possibility of detecting the WHIM in emission and constraining its thermal state with nextgeneration instrument we consider spectra in larger pixels $\left(2.6^{\prime} \times 2.6^{\prime}\right)$ similar to the CRIS angular resolution (goal). For this purpose we search for emission lines in each of the $128 \times 128 \times 7$ mock spectra using a two step procedure. (1) We identify the O VII (triplet) and O VIII lines by searching for local maxima in the appropriate energy range of the spectrum. (2) we compute the surface brightness of each line by summing the flux over energy bins redward and blueward of the line maximum and stop whenever the surface brightness $\mathrm{SB}_{t h}$ in the bin drops below $7 \times 10^{-3} \mathrm{ph} \mathrm{s}^{-1} \mathrm{~cm}^{-2} \mathrm{sr}^{-1}$ which is smaller than the typical line detection threshold for a 1 Ms observation with CRIS, as we will show $\S 3.2$. A simple example serves to clarify the procedure. To identify $\mathrm{O}$ VIII lines produced in the redshift range $z=[0,0.065]$ we search for local maxima in the energy range [0.613, $0.653 \mathrm{eV}$, where the lower bound is the redshifted energy of an O VIII photon emitted at $z=0.065$ The centroid of the line is identified with the maximum and its intensity is obtained by summing over all contiguous energy bins with surface brightness above that of the selected threshold. This strategy, which minimizes the chance contamination from gas at different redshifts, cannot be applied to real spectra since we lack the information of the redshift of the gas responsible for the line emission. This effect is discussed in $\S 6.1$.

Detectable WHIM lines are rare and it is unlikely to find two of them in the same mock spectrum. On the other hand, when approaching the surface brightness threshold, several weak emission lines start to appear. They are typically clustered around stronger lines and thus are likely to be physically associated to the same line emitting element. Considering all these lines would spoil line statistics since they would oversample the same line-emitting regions. To circumvent this problem we have adopted a simple criterion: we merge together all lines that are separated by a number of energy bins smaller than some minimum amount $n_{\text {bin }}$. In our analysis we have adopted $n_{\text {bin }}=8$ (corresponding $8 \mathrm{eV}$ ) after having checked that the number of lines with $0.1 \mathrm{ph} \mathrm{s}^{-1} \mathrm{~cm}^{-2} \mathrm{sr}^{-1}$ (strong enough to be hopefully detected by next-generation instruments) depends neither on $n_{\text {bin }}$ nor on $\mathrm{SB}_{t h}$.

To confirm that our B2 model fulfill the current constraints on the soft X-ray diffuse background, we have compared the total surface brightness predicted by the model in the $[0.65,1] \mathrm{keV}$ band with that measured by Hickox \& Markevitch (2007a) after removing the contribution from all known sources. According to the model, the WHIM contribution to the surface brightness contributed is $(3.6 \pm 0.3) \times 10^{-13} \mathrm{erg} \mathrm{cm}^{-2} \mathrm{~s}^{-1} \mathrm{deg}^{-1}$, safely below the observed value of $(1.0 \pm 0.2) \times 10^{-12} \mathrm{erg} \mathrm{cm}^{-2}$ $\mathrm{s}^{-1} \mathrm{deg}^{-1}$. The more conservative B1 model, which predicts a lower surface brightness is also obviously in agreement with observations.

\subsection{Line Statistics}

After having identified all O VII and O VIII emission lines in the mock spectra we can compute their cumulative number per unit redshift as a function of the line surface brightness. The top panel of Figure 2 shows the case of the O VII triplet whose surface brightness is the sum of the three lines. The black continuous curves refer to model B2 while the red dashed curves refer to model B1. For each model, the upper curve accounts for the gas emission from all gas particles along the line of sight and the lower curve considers only the contribution from the "WHIM phase" (see $\S 2.1$ ). In the B2 model the lines contributed by the warm hot gas constitutes a significant 
TABLE 1

EMISSION LINE DETECTIONS

\begin{tabular}{cccccccc}
\hline \hline Model & $t_{\text {exp }}$ & $f_{\text {line }}$ & $d N_{\text {OVII }} / d z$ & $d N_{\text {OVIII }} / d z$ & $d N_{\text {OVII }+ \text { OVIII }} / d z$ & $N_{\text {OviI }+ \text { OvIII per deg }^{2}}$ \\
\hline B2 & 1.0 & 0.07 & $4.2(3.1)$ & $2.6(1.9)$ & $2.4(1.6)$ & $639(426)$ \\
B2 & 0.1 & 0.48 & $2.1(0.6)$ & $1.5(0.5)$ & $1.1(0.2)$ & $293(53)$ \\
B1 & 1.0 & 0.07 & $3.3(1.6)$ & $2.1(1.0)$ & $1.8(0.7)$ & $479(186)$ \\
B1 & 0.1 & 0.48 & $1.4(0.03)$ & $1.1(0.05)$ & $0.7\left(<10^{-3}\right)$ & $186(0)$ \\
\hline
\end{tabular}

Note. - Column 1: WHIM model. Column 2: Exposure time (Ms). Column 3: Minimum line surface brightness required for a $5 \sigma$ detection $\left(\mathrm{ph} \mathrm{s}^{-1} \mathrm{~cm}^{-2} \mathrm{sr}^{-1}\right)$. Column 4: Expected number of $\mathrm{O}$ VII detections per resolution element and unit redshift Column 5: Expected number of $\mathrm{O}$ VIII detections per resolution element and unit redshift. Column 6: Expected number of simultaneous $\mathrm{O}$ VIII and $\mathrm{O}$ VIII detections per resolution element and unit redshift. Column 7: Expected number of simultaneous $\mathrm{O}$ VIII and O VIII detections per square degree due to gas within $z=0.5$. All estimates assume an angular resolution of $2.6^{\prime} \times 2.6^{\prime}$. The numbers in parenthesis indicate lines contributed by the WHIM.

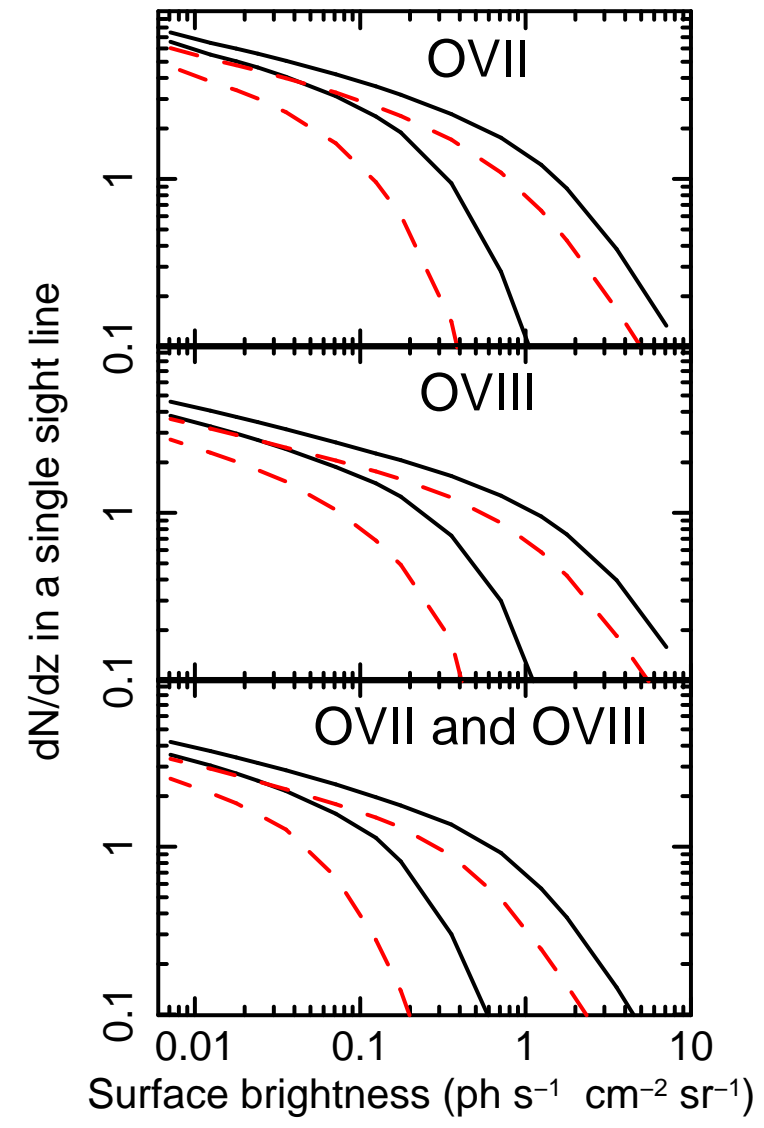

Fig. 2.- Cumulative number of emission lines per unit redshift as a function of line surface brightness. Each panel refers to O VII triplet (top), O VIII Ly $\alpha$ (middle) or both O VII triplet and O VIII Ly $\alpha$ (bottom). Black, solid curves indicate model B2, while red, dashed curves model B1. For each model, upper curve shows all the gas and lower curve refers to "WHIM phase" gas only.

fraction of the total. However, this fraction decreases with the surface brightness of the lines. It ranges from $\sim 100 \%$ for the weakest line to $\sim 5 \%$ for the brightest one. This result shows that the possibility of detecting the WHIM emission lines increases when decreasing the line detection threshold and clearly illustrates the need of increase the sensitivity of next-generation instruments. Model B1 predicts $\sim 30 \%$ less lines than model B2, as expected. The difference among the models is almost independent of the line surface brightness. For a reference line surface brightness of $0.1 \mathrm{ph} \mathrm{s}^{-1} \mathrm{~cm}^{-2} \mathrm{sr}^{-1}$ (match- ing the typical detection threshold for next-generation $\mathrm{X}$-ray spectrometers as we will show) the expected number of detected lines is of order of unity, indicating that the chance of multiple line detections in a pixel is rather small.

The middle panel of Figure 2 shows the cumulative $d N / d z$ statistics for the $\mathrm{O}$ VIII line. All considerations made for the O VII lines apply to the O VIII case except for the fact that, for a given surface brightness the expected number of O VII triplets is larger than that of O VIII lines. This difference becomes smaller if one considers the brightest O VII lines rather than the triplet. The bottom panel shows the cumulative $d N / d z$ of those line systems that exhibit both the O VII triplet and O VIII line as a function of the total surface brightness. The curves are similar to those of the O VIII lines (middle panel) except that at the bright end. This is due to the fact that presence of an O VIII line almost invariably guarantees that of an O VII triplet with similar (or larger) surface brightness.

Our results can be compared with the theoretical predictions of Cen \& Fang (2006) who used more sophisticated models that account for departures from ionization equilibrium and the effects of galactic superwinds. Their predicted cumulative number of emission lines (see Fig. 12 of the paper) is similar to that predicted by model B2. In particular the agreement is remarkable for the O VIII lines, while we seem to over predict that of O VII triplets. In addition, we note that the difference between model B1 and B2 is smaller than the scatter in their model predictions, indicating that we are being generous in our attempt of bracketing theoretical uncertainties.

\subsection{Expected WHIM detections}

To detect O VII and O VIII emission lines one needs to discriminate the genuine, weak emission signal from the astrophysical foreground and background which represents the main source of noise, typically exceeding the instrumental background. The problem of detecting the WHIM emission lines is analogous to that of detecting a low-surface brightness source with a typical angular extension is a few arcmin, i.e., the typical angular size of a WHIM filament at $z \sim 0.3$, The signal to noise is then optimized by measuring spectra with an angular resolution comparable to that of the sources over large FOV to detect as many WHIM lines as possible. In practice, one wants to perform spatially resolved spectroscopy over 
large areas with an angular resolution of few arcmin. The CRIS detector proposed for the Xenia mission and that we are considering as a prototype for next-generation spectrographs fulfills these requirements.

To investigate the possibility of detecting the WHIM through line emissions and assess the statistical significance of the detections one needs to quantify the noise which is mainly contributed by two sources: the cosmic $\mathrm{X}$-ray background (CXB, hereafter) produced by unresolved extragalactic point sources, and the foreground emission of our Galaxy.

The CXB is contributed by all X-ray sources (mostly AGN) below the source detection threshold. Its intensity depends therefore on the characteristics of the detector and on the exposure time. A conservative upper limit to the $\mathrm{CXB}$ can be obtained from the $\mathrm{XQC}$ rocket experiment (McCammon et al. 2002) since this estimate includes the contribution of all point sources in the FOV of $\sim 1 \mathrm{sr}$. In this case the CXB surface brightness is $f_{\mathrm{CXB}} \sim 30 \mathrm{ph} \mathrm{s}^{-1} \mathrm{~cm}^{-2} \mathrm{sr}^{-1} \mathrm{keV}^{-1}$. This value can be reduced by removing all resolved sources. One can remove sources previously detected in some other surveys. However, efficient removal would be possible only in areas covered by deep surveys, which are typically small. The alternative is to perform simultaneous deep imaging and resolved spectroscopy of the same areas, a strategy that would be possible if both a CCD-like imager and a 2D spectrograph with similar FOVs were available. This is precisely the case of of the Xenia mission concept which should be capable of resolving and removing $~ 1000$ AGN per square degree down to a limiting flux of $2.5 \times 10^{-16} \mathrm{erg} \mathrm{s}^{-1} \mathrm{~cm}^{-2}$ in the $0.5-2 \mathrm{keV}$ band. If AGNs were isotropically distributed, then a spectrograph with $\sim 4^{\prime}$ angular resolution would have all its pixels contaminated by AGN emission. Taking into account the angular clustering of the AGNs Vikhlinin \& Forman 1995), this contamination would affect $\sim 63 \%$ of the pixels if the angular resolution of the instrument is improved to $2.5^{\prime}$ (i.e., the CRIS goal). It is clear that the best compromise between low AGN contamination and large number of available pixels depends on the actual characteristics of the detector and on the availability of a CCD imager. For the sake of simplicity in this work we adopt the conservative solution of keeping all pixels and thus we assume $f_{\mathrm{CXB}}=30 \mathrm{ph} \mathrm{s}^{-1} \mathrm{~cm}^{-2} \mathrm{sr}^{-1} \mathrm{keV}^{-1}$.

The Galactic foreground is basically contributed by three sources: the so-called local bubble, the Galactic halo, and the solar wind charge exchange (SWCX). Following McCammon et al. (2002) we describe the foreground emission as the sum of absorbed thermal model (wabs and apec model in the XSPEC package respectively) and unabsorbed thermal model (apec). The former component represents the Galactic halo, while the latter the sum of local bubble and SWCX. One may think that this model is too crude, since the SWCX contribution, which is non thermal, is expected to outshine that of the local bubble. Moreover, the variable nature of the SWCX and the lack of strong observational constraints (e.g., Henley \& Shelton 2008; Fujimoto et al. 2007) make it difficult to model its contribution. However, for the purpose of this work, this approximation is reasonable for two reasons. First, it has been shown that the sum of two thermal components, although inaccurate, is quite effective in reproducing observations, at least within the

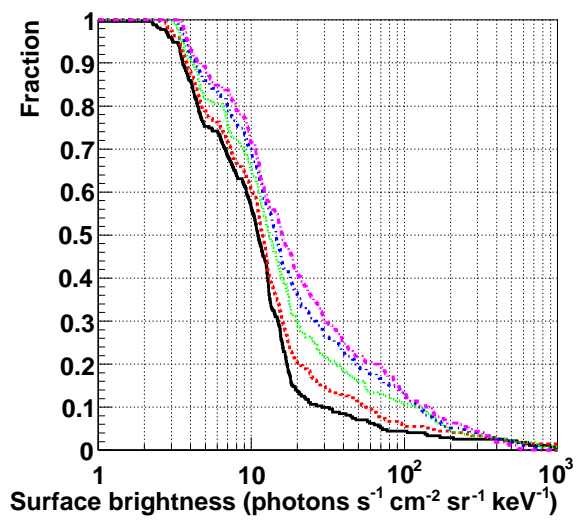

FIG. 3.- Fraction of energy bins (each bin with $1 \mathrm{eV}$ width) in which the measured surface brightness is above the value indicated on the $X$-axis. Different curves represent different energy resolution (FWHM) of the instrument: $1 \mathrm{eV}$ (red dashed), $3 \mathrm{eV}$ (green dotted), $5 \mathrm{eV}$ (blue dot-dashed), and $7 \mathrm{eV}$ (magenta dash dotted). The black, solid curve shows the case of no convolution with the detector response.

energy resolution of CCD detectors (Galeazzi et al. 2007; Yoshino et al. 2009). Second, in the analysis of the real spectra one ignores the energy ranges in which Galactic emission lines are present. These lines include $\operatorname{Ly} \beta, \operatorname{Ly} \gamma$, and $\mathrm{Ly} \delta$ transitions from $\mathrm{H}$-like $\mathrm{C}$ and $\mathrm{N}$ atoms, which should constitute strong features of the SWCX emission. In fact, all SWCX lines listed by Koutroumpa et al. (2006) and Bodewits et al. (2007) with a contribution of $>$ a few $\%$ of O VII triplets are in energy intervals that we ignore when we search for the WHIM lines in $\S 6.1$.

Since the energy spectrum of the Galactic foreground is characterized by prominent emission lines (see Fig. 2) and we are interested in identifying those produced by the WHIM, Galactic foreground flux cannot be estimated by the averaged flux over the wide energy range (e.g., $0.4-0.7 \mathrm{keV}$ ). More realistically, we would need to restrict the search to those energy bins that are not heavily contaminated by Galactic lines. Once again, when adopting this strategy one should compromise between the need of keeping as many energy resolution elements as possible and that of minimizing the Galactic signal. The solution can be found by looking at Figure 3 that shows the fraction of energy bins in which the measured foreground surface brightness is above the value indicated on the $X_{-}^{-}$ axis. The shape of the curves that refer to the case of no convolution with the detector response (black, solid) and of $1 \mathrm{eV}$ energy resolution (red, dashed) is characterized by a sharp drop at moderate surface brightness values, followed by a flattening in the high surface brightness tail. This bi-modality reflects the transition from a regime in which the flux is dominated by the continuum contribution (the low surface brightness end) to a regime dominated by the emission of bright lines (the high surface brightness end). The transition occurs at a surface brightness of $f_{\mathrm{FG}} \sim 20 \mathrm{ph} \mathrm{s}^{-1} \mathrm{~cm}^{-2} \mathrm{sr}^{-1} \mathrm{keV}^{-1}$, which therefore we take as the reference value for the Galactic foreground continuum signal in this paper. The other curves of Fig. 3 refer to different energy resolutions. As the energy resolution degrades, the fraction 
of high Galactic surface brightness becomes larger. The influence is considered in $\S 6.2$.

Having estimated the two noise sources $\left(f_{\mathrm{CXB}}\right.$ and $f_{\mathrm{FG}}$ to 30 and $20 \mathrm{ph} \mathrm{s}^{-1} \mathrm{~cm}^{-2} \mathrm{sr}^{-1} \mathrm{keV}^{-1}$, respectively) and ignoring the negligible contribution from the instrumental noise (Kelley et al. 2007), one can compute the significance of a line detection from the following expression:

$$
\sigma_{\text {line }}=\frac{f_{\text {line }}}{\sqrt{\left[f_{\text {line }}+\left(f_{\mathrm{CXB}}+f_{\mathrm{FG}}\right) \Delta E\right]}} \sqrt{\Delta \Omega t_{\text {exp }} A_{\text {eff }}},
$$

where $f_{\text {line }}$ is the source line flux in units of $\mathrm{ph} \mathrm{s}^{-1} \mathrm{~cm}^{-2}$ $\mathrm{sr}^{-1}, \Delta E$ is the energy resolution of the instrument, $A_{\mathrm{eff}}$ its effective area, $\Delta \Omega$ its angular size (or the pixel size in the case of this work), and $t_{\exp }$ is the exposure time. Eq. (11) assumes that both signal and noise can be treated as Poisson variables.

In Table 1 we list for the two WHIM model explored (column 1) and two different exposure times considered in this work (column 2), the minimum line surface brightness required for a $5 \sigma$ line detection calculated with Eq. (1) in a hypothetical observation performed with CRIS (col. 3). The corresponding number of detections per resolution element and unit redshift can be obtained from the $d N / d z$ statistics plotted in Fig. 2. They are listed in columns 4 (O VII), 5 (O VIII), and 6 (simultaneous $\mathrm{O}$ VII and $\mathrm{O}$ VIII detections). Column 7 shows the number of $\mathrm{O}$ VII+O VIII WHIM detections expected in the 1 degree $^{2}$ FOV due to the gas at $z \leq 0.5$. Each entry in columns $4-6$ shows two values. The higher one represents the expected number of detected lines contributed by all gas, not just in the WHIM phase. The smaller one, in parenthesis, refers to lines that can be attributed to the WHIM. All detection estimates assume an angular resolution of $2.6^{\prime} \times 2.6^{\prime}$ (corresponding to the sum of $2 \times 2$ pixels in the simulated dataset to the CRIS goal resolution) and $1 \mathrm{eV}$ energy resolution. These estimates do not account for the presence of spurious emission lines that contaminates $\sim 20 \%$ of the energy band analyzed. Their impact on the ideal detection estimates listed in Table 1 is estimated in $\S 6$

¿From Table 1 we see that the expected number of detections is quite large even in the least favourable case (model B1, $t_{\text {exp }}=100 \mathrm{ks}$, simultaneous O VII+O VIII detection). However, only $1 \mathrm{Ms}$ long observations guarantee to observe WHIM lines in the most conservative scenario of model B1. With the more realistic model B2 one expects to be able to detect WHIM lines also with shorter (100 ks) exposure times. We note that WHIM detection estimates in column 7 are to be regarded as conservative since they only consider gas out to $z=0.5$ whereas hydrodynamical simulations show that the WHIM mass fraction is still quite large at $z=1.0$. Overall, these results indicate that next-generation instruments will allow one to unambiguously detect the WHIM in emission and, as we will see in the next sections, to characterize its thermal state and trace its spatial distribution.

\section{PHYSICAL PROPERTIES OF THE WHIM IN EMISSION}

In this section we investigate the physical properties (density $\rho_{\text {gas }}$, temperature $T$, and mass $M$ ) of the WHIM that, according to our results, can potentially be detected by deep resolved spectroscopy in next-generation X-ray missions. The relevant quantities obtained from the anal- ysis of the mock X-ray spectra will be compared with the corresponding "true (input)" physical properties of the WHIM measured directly from the simulation. To perform realistic comparisons the "true" quantities were measured by averaging over all gas particles that contribute to the spectral signal, i.e. particles within volume elements. The data used for comparison are taken from the FOV of $5.5^{\circ} \times 5.5^{\circ}$ and the redshift range of $0.202<z<0.274$. The angular size of each volume element is $2.6^{\prime} \times 2.6^{\prime}$ (the angular resolution) in $\S 4.1$ and $1.3^{\prime} \times 1.3^{\prime}$ in $\S 4.2$. The depth of a volume element is 3 $\mathrm{Mpc}$ (comparable to the energy resolution).

\subsection{Density and temperature of detectable WHIM}

The thermal state of the gas can be appreciated from the phase-space diagram shown in top-left panel of Fig. 4. Colour contours are drawn in correspondence of the same gas mass fraction, indicated in the color-scale. This plot is analogous to the one shown in Fig. 6 of Branchini et al. (2009) with some important differences. First of all here we only consider particles with $T>10^{5} \mathrm{~K}$ that are potentially relevant for the line emission. Second, this plot refers to redshift slice $0.202<z<0.274$, considerably narrower than $0<z<0.2$, the one considered by Branchini et al. (2009). Finally, and most important, the plot refers to gas density and temperature averaged in the $2.6^{\prime} \times 2.6^{\prime} \times 3 \mathrm{Mpc}$ volumes and not to the density and temperature of each gas particle in the hydrodynamical simulation, which cannot be observed directly. The simulated data considered in Fig. 4 refers to the full FOV of $5.5^{\circ} \times 5.5^{\circ}$ but rather thin redshift slice to avoid large variation in the volume of the resolution elements. The smoothing effect induced by the volume averaging is very evident in the poor sampling of the high-density and high-temperature regions, when compared to Fig. 6 of Branchini et al. (2009). The effect can be further appreciated by looking at the black curve in the bottom panel of Fig. 4 which shows the probability distribution function of the gas density mass-weighted within each resolution element. The gas density on the $X$-axis is expressed in units of mean density. The distribution function, which was obtained by marking the phase-space plot over the gas temperature, looks remarkably symmetric. This symmetry is a result of the averaging process that deletes the high density tail that characterize the density distribution function of the gas particles (Davé et al. 2001).

The top right panel of Fig. 4 is analogous to the top left one but considers only gas elements for which the combined $\mathrm{O}$ VII and $\mathrm{O}$ VIII line surface brightness is above the $5 \sigma$ detection threshold of $0.07 \mathrm{ph} \mathrm{s}^{-1} \mathrm{~cm}^{-2}$ $\mathrm{sr}^{-1}$, line systems that could be detected with $1 \mathrm{Ms}$ observation with CRIS. This plot clearly indicates that next-generation instruments will preferentially probe the hottest part of the WHIM with $T>10^{6} \mathrm{~K}$ with typical density $\delta_{g} \sim 100$, hence confirming the results of Bertone et al. (2010).

This result is better illustrated by the red dotted curve in the bottom panel of Fig. 4 which is analogous to the black curve but only refer to the gas that can be potentially detected in a $1 \mathrm{Ms}$ observation. It clearly shows that detectable emission lines preferentially probe high density regions. The integral of the the red dotted curve shows that $30 \%$ of the total gas mass (the integral of 

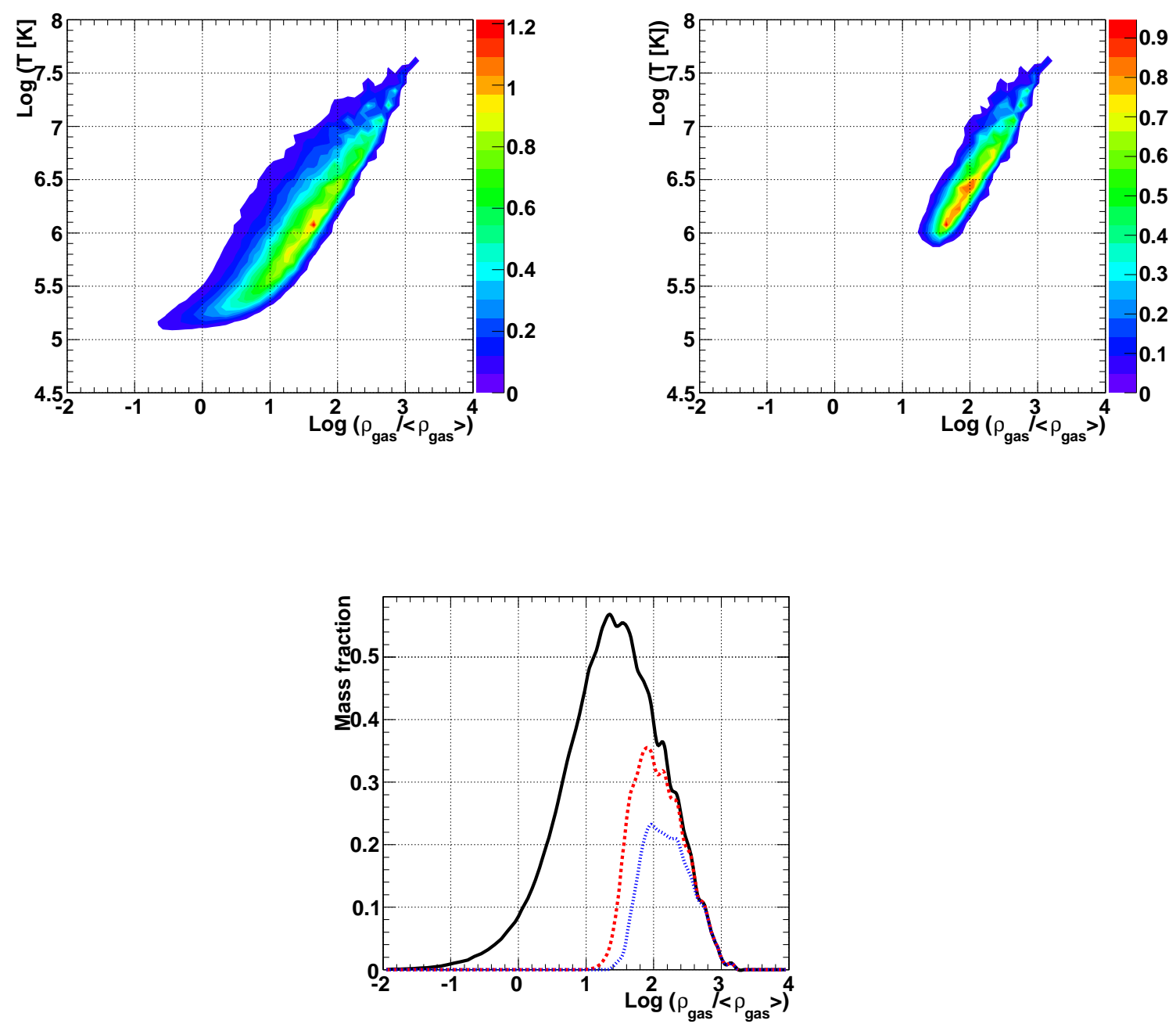

FIG. 4. - Top left: Contours of constant gas mass fraction in phase-space, i.e., the fraction in unit $\log \rho$ and $\log T$ intervals. The gas density is normalized to its cosmic mean ( $X$-axis) and its temperature is in unit of $\mathrm{K}$ ( $Y$-axis). The plots considers only gas particles in the redshift slice $0.202<z<0.274$ and with temperature $T>10^{5} \mathrm{~K}$. Color coded contours are drawn in correspondence of different values of the gas mass fraction, indicated in the color scale. Top right: same as the top-left panel, but referring to gas elements characterized by O VII + O VIII line systems strong enough to be detected with 1 Ms observation with CRIS. Bottom panel: the probability distribution function of the gas density obtained by merging the gas mass fraction in the $\rho$-T plane over its temperature. Black curve: all gas elements. Red dotted curve: gas elements in with both O VII and O VIII above $5 \sigma$ detection threshold of $0.07 \mathrm{ph} \mathrm{s}^{-1} \mathrm{~cm}^{-2} \mathrm{sr}^{-1}$, corresponding to $1 \mathrm{Ms}$ exposure with CRIS. Blue dashed curve: same as the red dotted curve but referring to a 100 ks exposure with detection threshold of $0.48 \mathrm{ph} \mathrm{s}^{-1} \mathrm{~cm}^{-2} \mathrm{sr}^{-1}$.

the black curve) could be probed via emission line spectroscopy. The remaining $70 \%$ of the gas that will go undetected typically resides in regions with density below 40 times the cosmic mean and constitutes the bulk of the WHIM. Reducing the exposure time to $100 \mathrm{ks}$ further decreases the fraction of detectable gas to $\sim 20 \%$ and shifts its mean density to larger values (blue dashed).

To further investigate the issue of the WHIM detectability, we plot in Fig. 5 the contours of gas mass fraction as a function of gas density ( $X$-axis) and line surface brightness $(Y$-axis). The two plots refer to the O VII triplet (left panel) and O VIII line (right panel), respectively, and consider all gas elements with $T>10^{5} \mathrm{~K}$ as in the top-right panel of Fig. 4. The first thing to notice is the very large range (about 10 order of mag- nitudes) of line surface brightness. Deep observations with a Xenia like mission will be capable of probing the gas above a detection limit of $\sim 0.1 \mathrm{ph} \mathrm{s}^{-1} \mathrm{~cm}^{-2} \mathrm{sr}^{-1}$. The expected line surface brightness spans $\sim 2$ order of magnitude when $\delta_{g} \gtrsim 100$. Below this value, and for a line surface brightness below the detection threshold, the iso-probability contours run almost parallel to the $Y$-axis, indicating the difficulty of detecting gas in lower density environment: huge improvements in the instrumental sensitivity would be required to probe the bulk of the WHIM in emission.

\subsection{Estimating the Gas Temperature}

The quantitative analysis of the mock spectra allows us to investigate the thermal properties of the emitting 

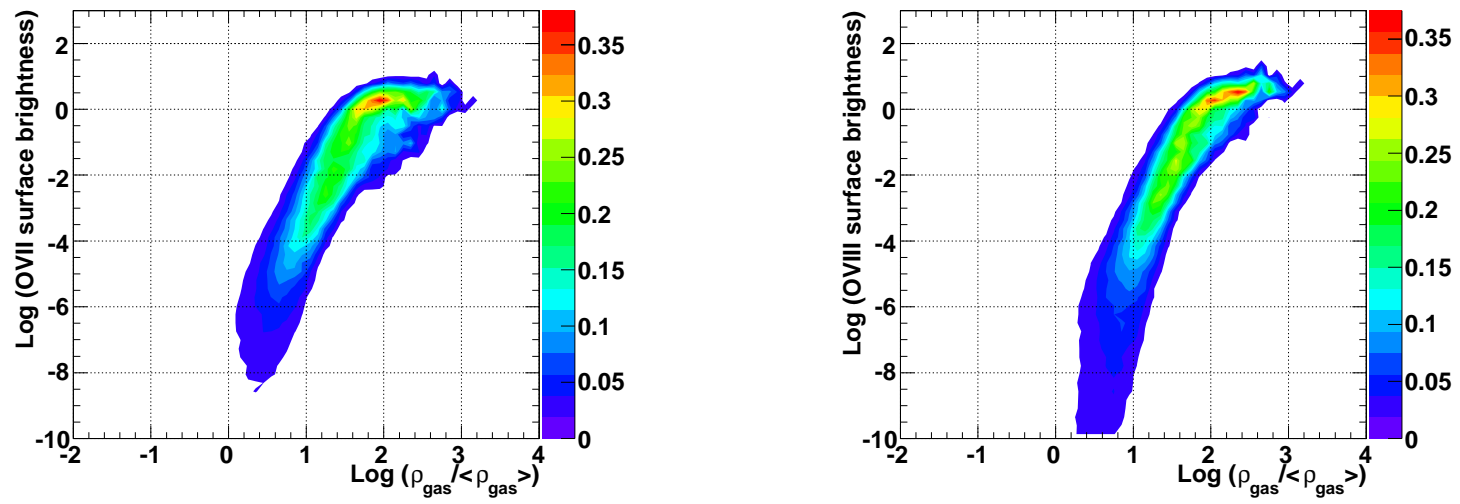

FIG. 5.- Contours of constant gas mass fraction in the line surface brightnesses-gas density plane. The plots considers only gas particles in the redshift slice $0.202<z<0.274$ and with temperature $T>10^{5} \mathrm{~K}$. The surface brightness $\left(Y\right.$-axis) is in units of ph s${ }^{-1} \mathrm{~cm}^{-2} \mathrm{sr}^{-1}$ and the gas density $(X$-axis) is normalized to its cosmic mean. Left panel: Surface brightness of the O VII triplet. Right panel : O VIII recombination line. The detection limit with a Xenia $1 \mathrm{Ms}$ observation is $0.07 \mathrm{ph} \mathrm{s}^{-1} \mathrm{~cm}^{-2} \mathrm{sr}^{-1}$, or $Y=-1.15$.

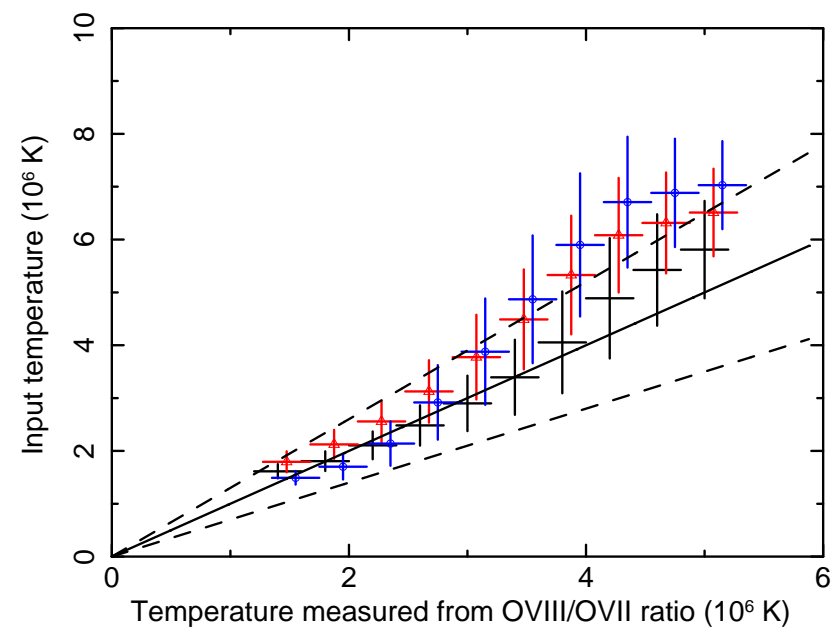

FIG. 6.- Relation between the gas temperature measured from the O VIII-O VII line intensity ratio in the mock WHIM spectra $(X$-axis) and different estimators for the underlying temperature of the gas ( $Y$-axis). The $Y$-axis of the black, red, and blue crosses refer to $\mathrm{O}$ VII-emission weighted temperature, $\mathrm{O}$ VIII-emission weighted temperature, and density weighted temperature, respectively. The data are taken from the FOV of $5.5^{\circ} \times 5.5^{\circ}$ and the redshift range of $0.202<z<0.274$. The angular size of each volume element $1.3^{\prime} \times 1.3^{\prime}$. Horizontal error bars correspond to the temperature bin size, while the vertical bars represent the scatter among resolution elements in the temperature bin. The perfect correlation (i.e., $Y=X$ ) is represented by the central solid line. The two external dashed lines represents $Y=1.3 X$ and $Y=X / 1.3$, respectively.

gas, namely to estimate the gas temperature. For those spectra in which both the O VII and O VIII lines have been detected one can use the line ratio to estimate the gas temperature. Alternatively one could estimate the gas temperature by comparing the relative intensity of the resonance, forbidden, and intercombination lines of the O VII triplet. These, however, can be detected only in the $\sim 20 \%$ of the cases. Therefore in this section we assess how well one can measure the gas temperature from the O VII-O VIII line intensity ratio when the CIE hypothesis holds true. For this purpose we have compared the temperature estimated from the mock spectra to the "true" temperatures of the gas responsible for line emission. The latter quantity, however, is not uniquely defined for two reasons. First, there is no one-to-one correlation between the measured quantity, which is derived from the line intensity, and the temperature of the gas particles. Second, the line emitting regions are sampled in finite volume elements because of the angular/energy resolution of the spectrometer. To tackle this problem we will use different estimators of the "true" temperature and compare the results with the temperature estimated from the $\mathrm{O}$ VII-O VIII line intensity ratio.

In Fig. 6 we have plotted the correlation between the temperature measured from the mock spectra $(X$ axis) and the one obtained from three different gas temperature estimators ( $Y$-axis). Here we consider the case of model B2 only. In the plot the different colors indicate the three different estimators: O VII-line-emission weighted temperature (black crosses) O VIII-line-emission weighted temperature (red crosses) mass-density weighted temperature (blue crosses). The "true" gas temperature is estimated in those resolution elements where the surface brightness of both O VII and $\mathrm{O}$ VIII is above $0.07 \mathrm{ph} \mathrm{s}^{-1} \mathrm{~cm}^{-2} \mathrm{sr}^{-1}$. The vertical size of each cross represents the scatter about the mean. The horizontal bar represents the size of the corresponding temperature bin.

The plot shows that a tight correlation between the measured and the "true" temperature is found with all estimators used. The temperature estimated from the O VIII-O VII line intensity ratio seems to best trace the $\mathrm{O}$ VII-emission weighted temperature of the gas, which is expected since temperature estimated from spectral lines preferentially weight those regions in which line emission is stronger. When the O VIII-emission weighted temperature is used the correlation is still tight but the slope is larger than unity. This is due to the fact that this estimator preferentially weights regions in which the O VIII is strong, i.e. regions in which the temperature is typically higher than those from which the O VII photons come from. With the mass-density weighted temperature the relation is unbiased for temperatures below $T \sim 3 \times 10^{6} \mathrm{~K}$ 
and then the correlation becomes steeper. This behavior can be understood as follows. The temperature measured from the spectra is sensitive to $T=(2-3) \times 10^{6} \mathrm{~K}$, where $\mathrm{O}$ VII and $\mathrm{O}$ VIII emissivity has a peak and it is reliably traced by the mass weighted temperature. At higher temperatures, a large fraction of the gas is too hot to contribute to the O VII emission and therefore it is not traced by the O VII line. As a result, the measured temperature underestimates the mass-weighted temperature of the gas.

Our assumption of CIE is well justified for gas in high density and temperature environments $\left(\delta_{g} \gtrsim 100\right.$ and $\left.T \gtrsim 2 \times 10^{6} \mathrm{~K}\right)$. Photoionization becomes non-negligible in regions characterized by lower density and temperature (i.e. when $\delta_{g} \lesssim 100$ and $T \lesssim 2 \times 10^{6} \mathrm{~K}$ ). The presence of the photoionizing background would change the ionization state of the gas. In particular, this background would ionize O VII into O VIII (Chen et al. 2003), and thus enhancing the intensity of the $\mathrm{O}$ VIII emission line relative to that of $\mathrm{O}$ VII. As a consequence, assuming the $\mathrm{O}$ VII/O VIII ratio of CIE overestimates the temperature of the gas. However, these effects will affect the gas properties in regions of moderate overdensity and temperature which could hardly be observed through emission line spectroscopy with current and next-generation detectors.

These results show that one can use the measured $\mathrm{O}$ VII and O VIII lines to estimate the temperature of the gas with a reasonable precision (10-20\% uncertainty, depending on the gas temperature). The measured quantity probes the temperature of the gas responsible for O VII emission and underestimates that of the O VIII emitting regions. The measured quantity also traces the temperature of the bulk of the gas for $T=(1-3) \times 10^{6} \mathrm{~K}$ and underestimates it in hotter regions.

Finally, we note that we have assumed a detector with angular resolution $1.3^{\prime} \times 1.3^{\prime}$ and that we do not consider the line broadening due to the detector response. The rationale behind this choice of the angular resolution is to reduce possible systematics in the estimate of the "true" temperature. In fact, using an angular resolution of $2.6^{\prime} \times 2.6^{\prime}$ as in the rest of the paper would not change appreciably the value of the measured temperature but would artificially reduce the estimated "true" temperature of the gas because of the increase in the filtering volume associated to the resolution element. A good angular resolution is thus important for an accurate determination of the physical state of the WHIM. Including the effect of the line broadening induced by the energy resolution of the detector would reduce the number of detectable line systems and would increase the statistical errors. However, we do not expect that these errors would seriously affect our temperature estimates since temperature errors are driven by the presence of multiphase gas within the resolution elements and not by the limited line statistics.

\section{PROBING THE SPATIAL DISTRIBUTION OF THE WHIM}

Spatially resolved spectroscopy provides the unique possibility of tracing the spatial distribution of the WHIM. In this section we use our simulated 2D spectra to investigate the 3D distribution of the WHIM responsible for detectable line emission. Here we focus our attention on the WHIM model B2 and assume a $1 \mathrm{Ms}$ observation with a CRIS-like spectrometer with angular resolution of $2.6^{\prime} \times 2.6^{\prime}$, energy resolution of $1 \mathrm{eV}$ and effective collecting area of $1000 \mathrm{~cm}^{2}$.

We only consider systems in which both O VII and O VIII lines can be detected at $\geq 5 \sigma$ significance and ignore the possibility of using additional metal lines associated to the source. The need of using both lines is dictated by the need of measuring the redshift of the associated line emitting region. This is guaranteed by selecting line pairs with energy ratio consistent with that of the O VII and O VIII lines. Using additional metal lines can, in principle, improve the identification of line emission systems and the determination of their redshift. However, the benefit in using these extra lines is in practice limited by the associated increase in chance contaminations. For example, a number of emission lines from Fe L complex can be seen in the mock spectra. However, these lines typically probe the hotter part of the WHIM, typically associated with virialized structure also responsible for strong continuum emission which reduces the significance of line detections. The Ne IX is a better signpost for the WHIM since it probes gas with $T \sim 10^{6.6} \mathrm{~K}$. However its emission line is too close to the Fe L lines to eliminate the risk of spurious detection. Finally, the energies of the redshifted $\mathrm{N}$ and $\mathrm{C}$ lines are typically found at the edges of the instrumental range, where a forest of emission lines due to $\mathrm{L}$ and $\mathrm{M}$ transition of heavier metals exists. For all these reasons we search the spectra for emission systems characterized by the O VII K $\alpha$ resonance and O VIII Ly $\alpha$ pair and do not look for additional ion lines.

Fig. 7 and 8 compare the 3D distribution of the selected O VII + O VIII line systems (lower panels) with the spatial distribution of the gas in the simulation (upper panels). Both figures show the same FOV of $5.5^{\circ} \times 5.5^{\circ}$, corresponding to a $6 \times 6$ mosaic with CRIS, but refer to two different redshift slice. Fig. 7 refers to the redshift interval $z=[0.2117,0.2317]$ corresponding to a $54 h^{-1} \mathrm{Mpc}$ slice at a comoving distance of $630 h^{-1} \mathrm{Mpc}$ in the cosmological model of the hydrodynamical simulation. Fig. 8 shows a much closer slice of $57 h^{-1} \mathrm{Mpc}$ at a comoving distance of $265 h^{-1} \mathrm{Mpc}$, corresponding to $z=[0.0805,0.1004]$.

In the gas map the blue regions correspond to isodensity surfaces drawn at $\rho_{\text {gas }} /\left\langle\rho_{\text {gas }}\right\rangle=75$. The green surfaces corresponds to regions characterized by smaller overdensity $\rho_{\text {gas }} /\left\langle\rho_{\text {gas }}\right\rangle=10$. At these overdensity levels the spatial distribution of the gas in the simulation is characterized by the well-known network of filaments punctuated by virialized structures that, in this iso-contour maps, appear as quasi-spherical "blobs". The filamentary structure is more clearly seen in the more distant slice thanks to the large volume sampled. The gas distribution in the nearby slice is clearly more dominated by a few, prominent structures, as expected.

The red regions in the bottom panels identify the positions of the selected O VII + O VIII line systems. Clearly these line systems qualitatively trace, in a sparse fashion, the large scale structures of the gas with overdensity $\rho_{\text {gas }} /\left\langle\rho_{\text {gas }}\right\rangle=75$. However the tracing is not unbiased since the line systems tend to undersample the more massive virial structures associated to the largest blob. This bias reflects the unfavorable ion balance for 

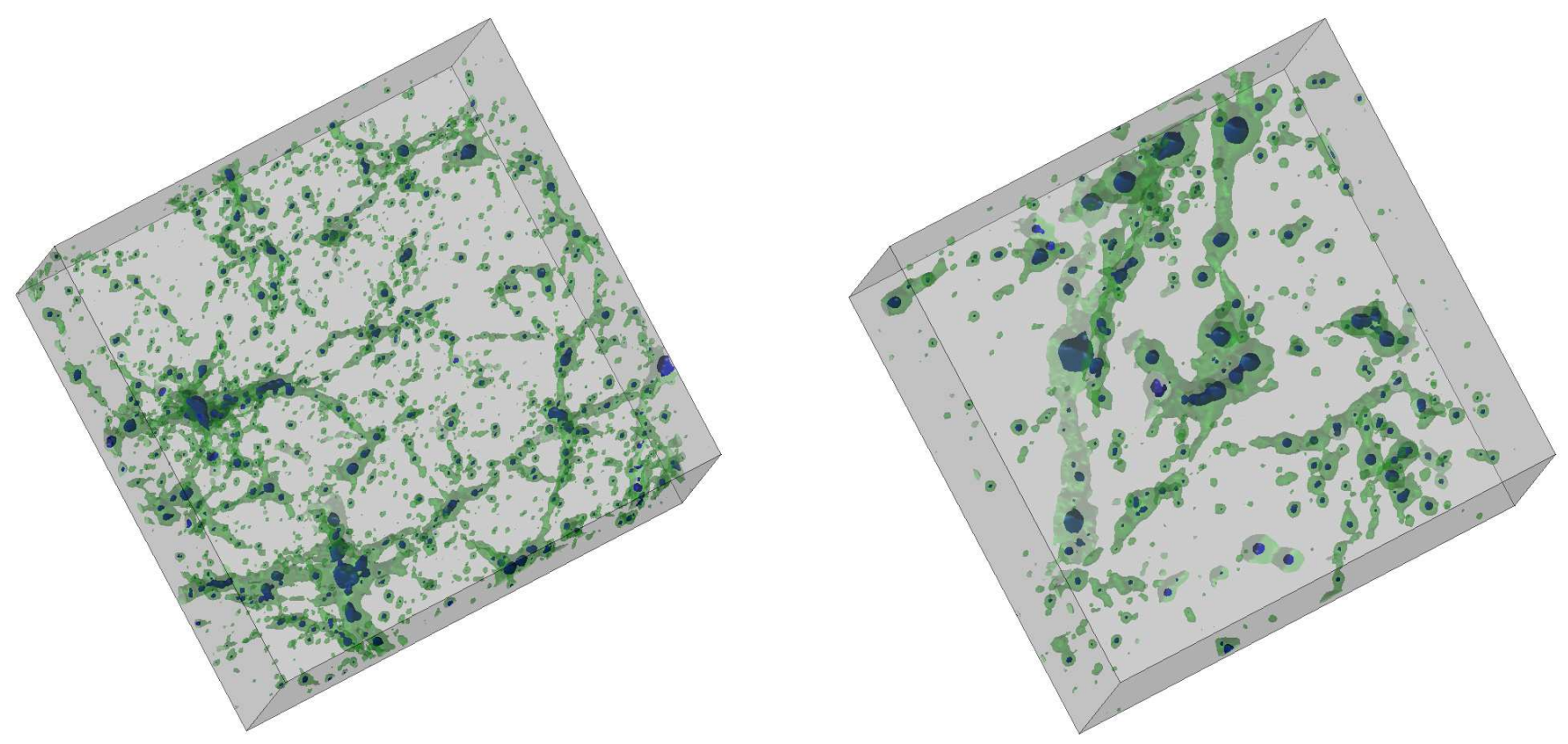

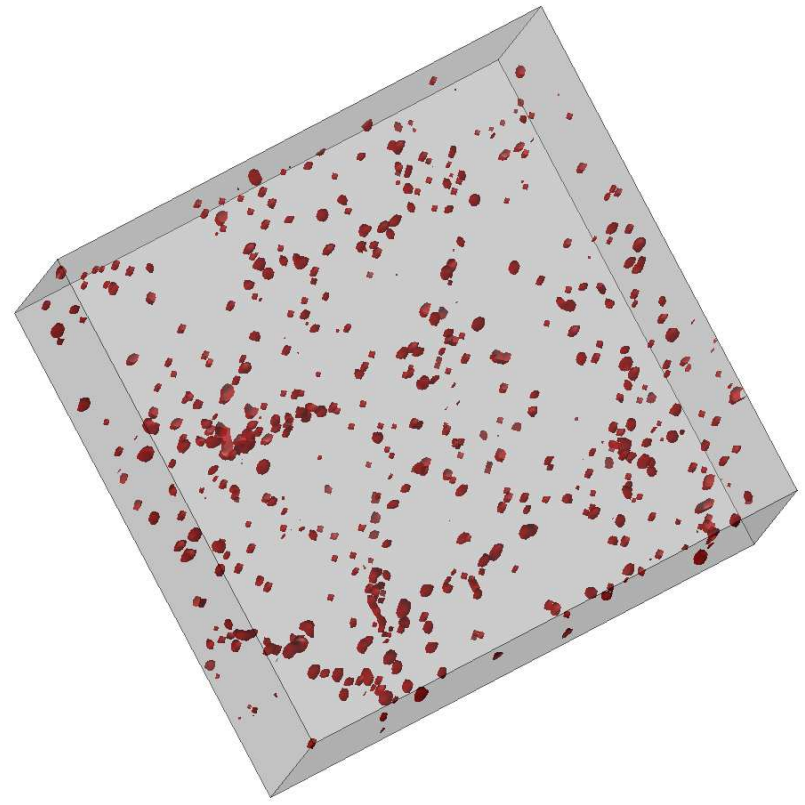

Fig. 7.- Slice of gas density contour (top) and O emission map (bottom) at $z=0.2117-0.2317$, corresponding to comoving distance of $603-657 h^{-1} \mathrm{Mpc}$. The field of view is $5.5^{\circ} \times 5.5^{\circ}$. The blue region of the top panel corresponds to $\rho_{\text {gas }} /\left\langle\rho_{\text {gas }}\right\rangle=75$, while the green region corresponds to $\rho_{\text {gas }} /\left\langle\rho_{\text {gas }}\right\rangle=10$. The red regions (blobs) show where both O VII and O VIII lines are identified with $>5 \sigma$ significance, with $1 \mathrm{Ms}$ exposure by Xenia CRIS-like instrument.

$\mathrm{O}$ VII and O VIII within hot virialized regions. The fact that $\mathrm{O}$ VII+O VIII line systems are poor tracers of gas at lower overdensity $\left(\rho_{\text {gas }} /\left\langle\rho_{\text {gas }}\right\rangle=10\right)$ confirms the fact that emission lines preferentially samples the fraction of the WHIM that resides in high density regions (Fig. 4).

This qualitative analysis confirms that next-generation X-ray spectrometers could be successfully used to trace the spatial distribution of the WHIM through its line emission. Thanks to the large number of the detected emission line systems, more quantitative analysis can be performed through statistical estimators. In

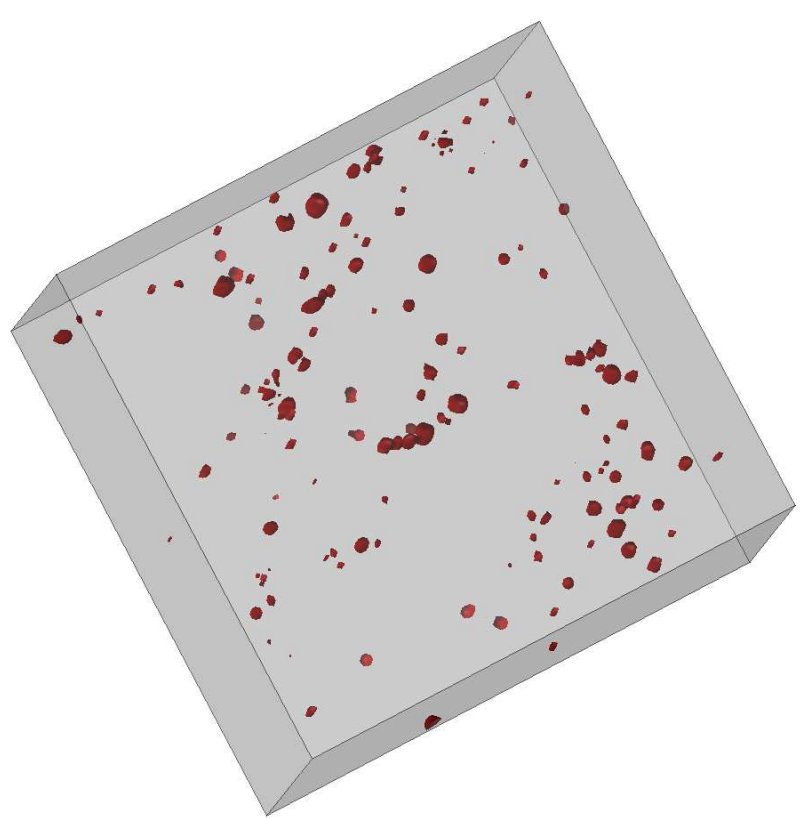

Fig. 8. - Same as Figure 7 but slice at $z=0.0805-0.1004$, corresponding to comoving distance of $237-294 h^{-1} \mathrm{Mpc}$. The field of view is $5.5^{\circ} \times 5.5^{\circ}$.

Ursino et al. (2010a), which uses the same mock spectra, it is shown that the two-point spatial correlation function of the line-emitters could be measured with a good accuracy. Its slope and correlation length, which should be similar to those of the galaxies, could be estimated at a few \% accuracy level.

The tracing of underlying gas by the detected line systems, which in the plots looks quite patchy, is possibly improved by adopting some adaptive smoothing technique like those based on the Wavelet transform analysis. The improvement would be particularly noticeable for nearby structures since, in this cases, the angular size of the associated gas cloud is larger than the angular resolution of the instrument. 


\section{ROBUSTNESS TESTS AGAINST NON-IDEAL EFFECTS}

In this section we assess the impact of foreground emission, energy resolution and contamination from spurious metal lines on the detectability of the extragalactic O VII and O VIII lines and on our capability of tracing the spatial distribution of the WHIM.

\subsection{Impact of contamination from spurious lines and high continuum}

We address the problem of identifying $\mathrm{O}$ VII and O VIII lines with no a priori knowledge of the nature of the detected lines and of their redshift. For this purpose the line-detection procedure have been modified from that depicted in $\S 3.2$. The rationale behind this choice is to avoid contamination from lines associated to different line emitting regions along the line of sight and to account for the fact that the line detection efficiency is not constant across the FOV since the level of the continuum emission depends on the direction. We have decided to ignore additional metal lines associated to the WHIM since their search would increase the chance of false identifications. To identify the putative WHIM emission systems we do not perform spectral fitting assuming some particular model but we search for the O VII $\mathrm{K} \alpha$ and O VIII Ly $\alpha$ line pairs with a strategy more conservative than that adopted in $\S 3$ and designed to account for the variable spectral continuum and for the presence of spurious spectral features. The procedure is as follows. (1) We deal with the fact that the continuum level may vary along the spectrum and also from spectra to spectra, across the FOV. To account for this effect we modify the line detection criterion: instead of setting a fixed line detection threshold, corresponding to a minimum line surface brightness, we define the detection significance $S$ by comparing the number of photon counts in $1 \mathrm{eV}$ bin (Counts) with the number of continuum counts (Continuum) obtained by averaging over the neighboring $50 \mathrm{eV}$ that do not contain line features above $2 \sigma$ or known galactic lines brighter than $>0.05 \mathrm{ph} \mathrm{s}^{-1} \mathrm{~cm}^{-2}$ $\mathrm{sr}^{-1} \mathrm{eV}^{-1}$. The significance is then defined as:

$$
S / N=\frac{\text { Counts }- \text { Continuum }}{1.0+\sqrt{\text { Continuum }+0.75}}
$$

where the denominator is an approximate $1 \sigma$ Poisson error (Gehrels 1986). Note that Eq. (2) is a similar quantity as Eq. (1) but is a quantity calculated with observed quantities. (2) We consider only lines in the energy range $[375,653] \mathrm{eV}$ detected at $5 \sigma$ significance (i.e., $S / N>5$ ). With this criterion we identify $7.8 \times 10^{3}$ lines $\mathrm{deg}^{-2}$ corresponding to transitions of highly ionized ions of $\mathrm{N}, \mathrm{O}, \mathrm{Ne}$, and Fe. (3) We minimize the chance of contamination by spurious lines by enforcing a number of constraints. (i) we reject all lines with flux of $>0.05 \mathrm{ph} \mathrm{s}^{-1} \mathrm{~cm}^{-2} \mathrm{sr}^{-1}$ $\mathrm{eV}^{-1}$ that could be attributed to Galaxy emission, i.e., all known Galactic lines; (ii) to avoid oversampling the same structures we discard all lines with energy within $15 \mathrm{eV}$ from that of a stronger emission line; (iii) we search for line pairs from the same angular element, and pick up the pairs only if the energy interval of the two lines is the same as that of redshifted $\mathrm{O}$ VII $\mathrm{K} \alpha$ resonance and O VIII Ly $\alpha$ lines. The emission lines without an associated O VII or O VIII line are discarded.
Using the procedure, we only consider those systems that both the O VII $\mathrm{K} \alpha$ and the O VIII Ly $\alpha$ lines are identified with significance larger than $5 \sigma$. As a result we obtain $446 \mathrm{deg}^{-2}$ line emission systems identified. This number is $\sim 30 \%$ less than those identified in $\S 3$. The discrepancy comes from the combined effect of line contamination and high continuum level. We confirm that, with this procedure, the contamination of chance coincidence of either O VII or O VIII lines with heavier metal lines is less than 10\%. Our spectra do not include photons emitted by gas at $z>0.5$. O VII lines emitted beyond this redshift would be redshifted out of the energy range probed by the detector, so the only impact of high redshift gas would be to increase the probability of contamination by higher energy lines such as O VIII, Ne IX, O X and Fe L complex. However, we do not expect a severe additional contamination for two reasons. First, the dimming of the emission line intensity, which scales as $(1+z)^{-3}$, is not exactly counterbalanced by the increase in the probed volume since,the latter decreases more slowly than $\propto(1+z)^{3}$ at high redshifts Second, at higher redshifts the mass fraction of the gas in the Ly$\alpha$ forest increases while the abundance of the gas contributing to O VII and O VIII lines emission decreases. To test the validity of these hypotheses and quantify line contamination by high-redshift gas we have created and analyzed mock spectra that accounts for emission by the gas out to $z<1.2$ and within a FOV of $2.6^{\circ} \times 2.6^{\circ}$ and have compared the results with the reference case of $z<0.5$. Including the emission from gas beyond $z=0.5$ decreases the number of detectable $\mathrm{O}$ VII-O VIII pairs by only $10-15 \%$.

\subsection{Impact of energy resolution}

A degradation in the energy resolution of the instruments affects the possibility of detecting the WHIM lines in two ways. First, it decreases the signal-to-noise ratio in Eq. (11). As a consequence, one needs to increase the surface brightness threshold for $5 \sigma$ detections, hence reducing the total number of detected lines. Second, with a large $\Delta E$ the chance of contamination from neighboring lines increases. Here we let the energy resolution vary in the range $[1,7] \mathrm{eV}$ which encompasses the values already achieved with current instrument $(\Delta E=7 \mathrm{eV}$ with Suzaku; Kelley et al. 2007) and the energy resolutions expected by currently planned X-ray missions equipped with a microcalorimeter spectrograph.

A visual impression of the cumulative impact of these two effects is given by Fig. 9, which shows a portion of the mock emission spectrum displayed in Fig. 11 but observed with different energy resolutions and in log scale. With $1 \mathrm{eV}$ resolution (FWHM), O VII triplets and O VIII resonant line at $z=0.033(0.55 \mathrm{keV}$ and $0.63 \mathrm{keV}$, respectively) are clearly detected, with a marginal detection of a emission line at $0.47 \mathrm{keV}$. With $\Delta E=3 \mathrm{eV}$, the $0.47 \mathrm{keV}$ line is below detection threshold, and with $\Delta E=5 \mathrm{eV}$ the $\mathrm{O}$ VII triplet becomes hard to observe because of the local $(z=0) \mathrm{O}$ VII emission. When $\Delta E$ is $7 \mathrm{eV}$, the $\mathrm{O}$ VII triplet drops below detection threshold.

A quantitative assessment of the contamination by spurious lines is provided by Fig. 3. The fraction of energy bins with surface brightness above that of the Galactic foreground emission $\left(f_{\mathrm{FG}}=20 \mathrm{ph} \mathrm{s}^{-1} \mathrm{~cm}^{-2}\right.$ $\mathrm{sr}^{-1} \mathrm{keV}^{-1}$ ) steadily increases with $\Delta E$ : from $20 \%$ for 
TABLE 2

NUMBER OF EMISSION LINE DETECTIONS WITH DIFFERENT DETECTOR ENERGY RESOLUTION

\begin{tabular}{ccccc}
\hline $\begin{array}{c}\Delta E \\
(\mathrm{eV})\end{array}$ & $\begin{array}{c}f_{\text {line }} \\
\left(\mathrm{ph} \mathrm{s}^{-1} \mathrm{~cm}^{-2} \mathrm{sr}^{-1}\right)\end{array}$ & $\begin{array}{c}d N_{\text {OVII }+ \text { OVIII }} / d z \\
\cdots\end{array}$ & $\begin{array}{c}\text { Fraction of } \\
\text { high } f_{\mathrm{FG}}(\%)\end{array}$ & $\begin{array}{c}N_{\text {OVII+OVIII per deg }}{ }^{2} \\
\text { in the simulation }\end{array}$ \\
\hline $\mathrm{-}^{\mathrm{a}}$ & 0.07 & 2.4 & 14 & 446 \\
1 & 0.07 & 2.4 & 20 & 396 \\
3 & 0.11 & 2.1 & 30 & 351 \\
5 & 0.13 & 2.0 & 36 & 274 \\
7 & 0.15 & 1.9 & 41 & 163
\end{tabular}

Note. - Column 1: Detector energy resolution. Column 2: Minimum line surface brightness required for a $5 \sigma$ detection $\left(\mathrm{ph} \mathrm{s}^{-1} \mathrm{~cm}^{-2} \mathrm{sr}^{-1}\right)$. Column 3: Expected number of simultaneous O VIII and O VIII detections per resolution element and unit redshift. The foreground emission is assumed to have a constant surface brightness of $20 \mathrm{ph} \mathrm{s}^{-1} \mathrm{~cm}^{-2} \mathrm{sr}^{-1} \mathrm{keV}^{-1}$. Column 4: Fraction of the energy range where foreground emission surface brightness exceeds $20 \mathrm{ph} \mathrm{s}^{-1} \mathrm{~cm}^{-2} \mathrm{sr}^{-1} \mathrm{keV}^{-1}$. Column 5: Number of simultaneous O VIII and O VIII detections per square degree in the mock spectra. All estimates assume an angular resolution of $2.6^{\prime} \times 2.6^{\prime}$ and model B2.

a Not convolved with detector energy resolution. The bin size is $1 \mathrm{eV}$.

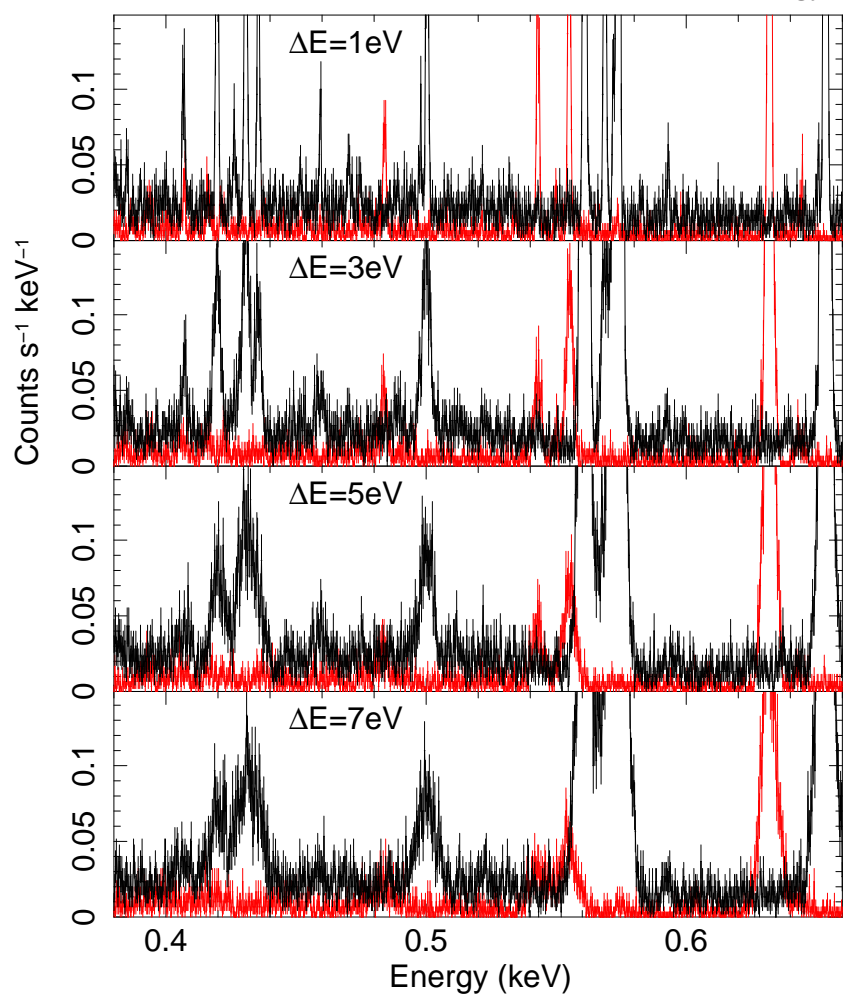

FIG. 9. - Mock spectrum in a $2.6^{\prime} \times 2.6^{\prime}$ area from a $1 \mathrm{Ms}$ exposure with Xenia CRIS. The black spectra are the sum of Galactic foreground and unresolved extragalactic background emission, while the red is extragalactic diffuse gas. The four panels of the each plot show the spectra convolved with the energy resolution (FWHM) of $1 \mathrm{eV}, 3 \mathrm{eV}, 5 \mathrm{eV}$, and $7 \mathrm{eV}$, respectively.

$\Delta E=1 \mathrm{eV}$ to $41 \%$ for $\Delta E=7 \mathrm{eV}$. In Table 2 we quantify how energy resolution affects the number of expected detections, splitting its effect in two: the increase of the surface brightness detection threshold (column 3) and the increase in the number of bins contaminated by the Galactic Foreground (column 4). All estimates assume the same setup as in Table 1 apart from energy resolution: WHIM model B2, 1 Ms observation, and 2.6 $\times 2.6^{\prime}$ angular resolution. The first effect is rather minor and reduced the expected number of line detection only by
$<20 \%$. On the other hand, contamination by galactic foreground has a more serious impact since the number of expected detection is directly proportional to that of the uncontaminated energy bins. Our results show that decreasing $\Delta E$ from 1 to $7 \mathrm{eV}$ reduces the number of expected detection by a factor of 2 . It should also be noticed that this effect does not randomly affect all emission line systems. The systems that are more seriously affected are those with redshifted emission lines that coincide with those of the Galactic foreground. This induces a non-trivial selection effect in the redshift distribution of the WHIM emitters that need to be corrected for to trace their 3D distribution.

To quantify the impact of the energy resolution on the expected number of WHIM line detections we have repeated the analysis performed in $\S 6.1$ after convolving the mock spectra with the appropriate response matrix. In the analysis of the response-convolved spectra, we again do not fit the spectra with a particular model, but searched for the O VII and O VIII line pairs. The only difference is that in the new analysis photon counts in neighboring energy bins are summed up before searching for emission lines. This is done to collect photons that are spread into several energy bins (the bin width is $1 \mathrm{eV}$ ) due to the energy resolution of the instrument. The results of this procedure obviously depend on the number of neighboring bins to be summed upon. We set this free parameter by maximizing the number of detectable emission lines. It turns out that the best results are obtained when summing over $((\Delta E / 1 \mathrm{eV})+1)$ bins for $\Delta E \leq 5 \mathrm{eV}$ and over $((\Delta E / 1 \mathrm{eV})-3)$ for $\Delta E=7 \mathrm{eV}$. The change of the trend for $\Delta E=7 \mathrm{eV}$ reflects the fact that at low energy resolution foreground contamination becomes the limiting factor. The number of expected detections is listed in column 5 of Table 2. It is clear that an energy resolution $\Delta E \leq 3 \mathrm{eV}$ is desirable, although even with $\Delta E=7 \mathrm{eV}$ one still expects $160 \mathrm{O} \mathrm{VII}+\mathrm{O} \mathrm{VIII}$ line detections per square degree. The reason why the number of expected detection of the 'no convolution' case is different from the analogous one listed in Table 1 is because in the line detection strategy used in this case, and described in $\S 6.1$, we have ignored the energy range in which the surface brightness of the foreground emission 
is above $f_{\mathrm{FG}}$. These ranges could be also be searched for detectable lines by locally increasing the detection threshold. However the expected increase in the number of detections is small since the intensity of the foreground emission lines is typically an order of magnitude larger than that of the WHIM lines.

\section{DISCRIMINATING AMONG WHIM MODELS}

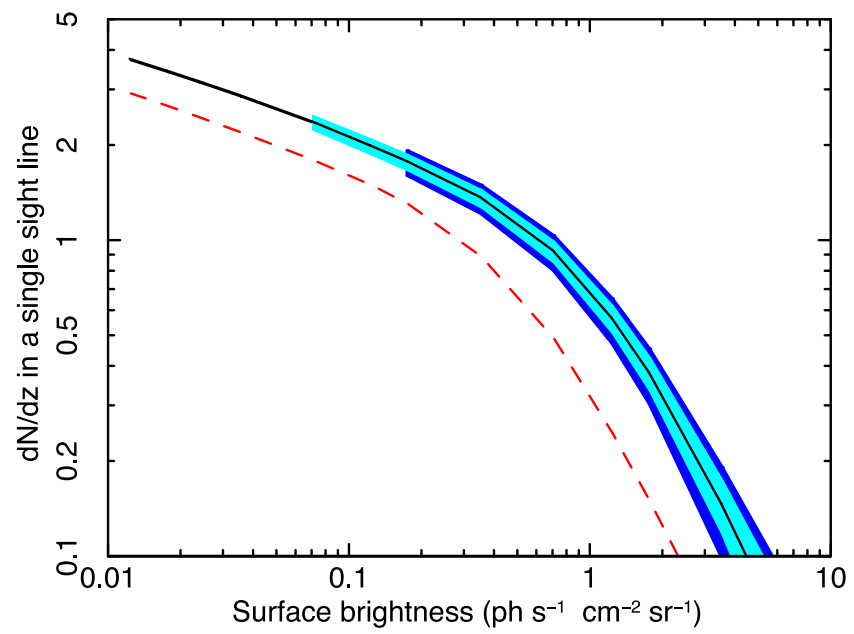

FIg. 10.- The expected $d N / d z$ curve with a Poisson error with $1 \mathrm{Ms}$ exposure, $2.6^{\prime} \times 2.6^{\prime}$ angular size, and $1 \mathrm{deg}^{2}$ FOV with CRIS. The black curve is the model curve (model B2). Color-filled areas are $1 \sigma$ Poisson error regions: cyan and blue are without considering detector energy resolution and with convolution with $7 \mathrm{eV}$ energy resolution, respectively. Red dashed line is from model B1 (Poisson error is not shown).

As discussed in $\S$ 2, current limitations in our understanding of the stellar feedback, chemical evolution, and metal diffusion process are reflected in the large uncertainties of the WHIM models. The most recent hydrodynamical simulations of the intergalactic gas at low redshifts have been used to construct different WHIM models by assuming different, physically plausible, recipes for the above processes. Comparisons between model predictions and observations can therefore constrain the cosmic history of chemical evolution and metal diffusion along with the underlying stellar feedback processes by discriminating among these WHIM models. UV observations are leading the way, since they currently provide the most stringent constraints on the WHIM models through O VI line statistics. X-ray observations can add additional constraints, through line statistics, direct assessment on the physical state and spatial distribution of the emitting gas.

In this section we assess to what extent future experiments will enable us to discriminate among different WHIM models. Here we focus on the $d N / d z$ statistics of $\mathrm{O}$ VII and O VIII lines. Fig. 10 shows the expected number of $\mathrm{O} \mathrm{VII}+\mathrm{O}$ VIII detection per unit redshift in an angular resolution element of $2.6^{\prime} \times 2.6^{\prime}$ and for $1 \mathrm{Ms}$ observation with CRIS for model B2 (black, continuous curve) and B1 (red, dashed). The cyan-colored band around the black lines show the $1 \sigma$ Poisson uncertainties for model $\mathrm{B} 2$ from the finite number of detections expected in a $1 \mathrm{deg}^{2}$ FOV. The expected errors are much smaller than the difference between model B1 and B2, suggesting that observations will efficiently discriminate among different WHIM models. The capability of constraining the physical processes that regulate the WHIM is further illustrated by pointing out that difference between model B1 and model B2 is of the same order as that between the WHIM models proposed by Cen \& Fang (2006), which adopt different prescription for the ionization balance and for galactic superwinds. The larger, blue uncertainty strips that extend beyond the surface brightness threshold of $0.15 \mathrm{ph} \mathrm{s}^{-1} \mathrm{~cm}^{-2} \mathrm{sr}^{-1}$ refers to the case of $\Delta E=7$ $\mathrm{eV}$. It shows that even with a rather modest energy resolution the discriminatory power of the $d N / d z$ test is still considerably large. The significance of discriminating the two models is $3-5 \sigma$ with a 1 deg $^{2}$ observation, depending on the energy resolution.

It should be noted that the cosmic variance introduces an additional error in determining the $d N / d z$ curve. We have estimated the contribution of cosmic variance by splitting the simulated $5.5^{\circ} \times 5.5^{\circ}$ field into several smaller regions. The variance in detectable emission line systems is $20 \%, 15 \%$, and $10 \%$, for the FOV of $0.25 \mathrm{deg}^{2}$, $1.0 \mathrm{deg}^{2}$, and $4.0 \mathrm{deg}^{2}$, respectively. Hence, a large-field mapping with $>4.0 \mathrm{deg}^{2}$ is desirable to make the uncertainty due to cosmic variance smaller than the statistical error ( $\sim 10 \%$ for $7 \mathrm{eV}$ energy resolution). This can be easily achieved with a dedicated mission such as EDGE and Xenia thanks to the large FOV.

Finally, we point out that emission-line studies provide better statistics than absorption studies because of the comparatively larger number of line detections. In fact, the two approaches are very complementary. Emission studies preferentially probe regions of enhanced density where the impact of stellar feedback is larger and chemical enrichment is presumably stronger. On the contrary, absorption studies preferentially probe regions of moderate overdensity where the bulk of the WHIM resides and thus are best indicated for closing the baryon budget at $z=0$. Space missions like EDGE, Xenia, and ORIGIN are designed to perform both emission and absorption analyses thanks to their capability of fast repointing that will enable us to use Gamma-ray bursts as distant beacon and search for the characteristic absorption features of the intervening WHIM Branchini et al. 2009).

Constraining abundance and physical properties of the WHIM from observations it is not straightforward. The first reason is that in order to trace the thermal history of the WHIM it requires an accurate and selfconsistent treatment of metal line cooling which, instead, is ignored in the present work. The second reason is the approximate treatment of a number of processes and quantities (galactic winds, AGN feedback, stellar IMF and sub-resolution turbulence; see Bertone et al. 2010; ?; ?; Schave et al. 2010) that may affect the thermal state of the WHIM in the current numerical experiments. Some of these processes have little impact on observable quantities such as O VIII surface brightness (e.g., Bertone et al. 2010), and hence it cannot be used to discriminate among competing WHIM models. On the other hand, emission line statistics and analysis will no doubt help to lift some of the model degeneracy, especially when coupled to observational constraints on the spatial distribution of the line-emitting gas, like those derived from the estimate of the angular and spa- 
tial two-point correlation function of the O-line emission (Ursino et al. 2010a).

\section{DISCUSSION AND CONCLUSIONS}

In this work we have analyzed the mock X-ray spectra extracted from the the hydrodynamical simulation of Borgani et al. (2004) to investigate the possibility of detecting the WHIM in emission with next-generation Xray satellites, assess its thermal state and trace its spatial distribution. Our theoretical predictions are based on the WHIM model of Branchini et al. (2009). The theoretical predictions presented in this work are mainly based on their model "B2" although we also consider the more conservative (and less realistic) WHIM model "B1" to bracket theoretical uncertainties. Finally, the experimental setup considered here is the same one proposed for the Xenia mission. In particular we will assume that our mock spectra were "observed" with the imaging spectrometer CRIS.

We showed that a CRIS-like spectrograph will enable us to detect a large number of O VII+O VIII line emitting systems, to estimate their temperature from the line ratio and to trace their spatial distribution. The line systems are expected to trace the hotter and denser part of the WHIM, typically associated to the outskirts of large virialized structures. Our results suggest that the number of expected detections is large enough to discriminate among different WHIM models and to constrain the physical processes that regulates its thermal state and metal content, even when one accounts for contamination effects and allows for a moderate $(\sim 7 \mathrm{eV})$ energy resolution.

All the results presented in this work are based on a WHIM model that fulfill all observational constraints but is nevertheless poorly constrained by observations. From the theoretical side, WHIM models suffer from the limited knowledge of the feedback mechanisms and metal diffusion processes that potentially affect the WHIM properties. In particular, the WHIM model adopted here, though effective in describing observations, lacks self-consistency since the metallicity of the gas is assigned in the post-processing phase rather than being computed during the run. To fix these problems one should modify theoretical prescriptions for feedback process or metal diffusion and gas cooling. Let us asses qualitatively the impact of having ignored line cooling. Efficient metal cooling requires a cooling time $t_{\text {cool }}$ shorter than the Hubble time $t_{\mathrm{H}}$. With no metal cooling, $t_{\text {cool }}<t_{\mathrm{H}}$ only when $\delta_{g}>1000$. Cooling become more efficient in presence of metals and less efficient with the gas temperature, i.e. $t_{\text {cool }}$ decreases with metallicity (e.g., Figure 4 of ?), and increases with $T$. On the other hand, we have shown that emission line spectroscopy can only detect gas with $T>10^{6} \mathrm{~K}$ (Figure 4). Taking all this into account and assuming a reference metallicity of $\sim 0.1 Z_{\odot}$ one finds that $t_{\mathrm{cool}}<t_{\mathrm{H}}$ if $\delta_{g}>300$ for $T=10^{6} \mathrm{~K}$ and if $\delta_{g}>1000$ for $T=2 \times 10^{6} \mathrm{~K}$. This simple arguments indicate that metal line cooling preferentially affects dense $\left(\delta_{g}>300\right)$ gas typically associated to galaxy groups rather than the WHIM. Indeed, inefficient cooling in the simulation is probably the cause of the excess X-ray emission from groups in the simulation.

In fact, the impact of the metal cooling is likely to be more significant because it affects the whole thermal his- tory of the baryons (????). When metals are transferred from galaxies, either by galactic wind or AGN, both the density and metallicity are high, and hence metal cooling is very efficient. After the temperature is lowered by metal cooling, the gas is further cooled down by adiabatic expansion. As a result, the thermal state of baryons at $z \sim 0$ is determined by their thermal history. The impact of these effects can only be assessed by running a numerical simulation in which metal cooling is accounted for self-consistently and, more importantly, in which the relevant physical process are included correctly. However, current numerical experiments which include a self consistent treatment of metal cooling (Bertone et al. 2010) seem to corroborate our qualitative argument since they show that metal cooling mostly affect regions with overdensity typical of galaxy groups, rather than the WHIM. For this reason, we are quite confident that considering predictions from both model B2 and the very conservative model B1 will allow us to bracket theoretical uncertainties, including incorrect treatment of metal cooling in the numerical simulation.

Bertone et al. (2010) have shown that predictions on the detectability of the $\mathrm{O}$ VIII line are are quite robust to the different model prescription with the exception of the cooling function. They show that using a metal-dependent cooling instead of a metal-independent scheme (as in our model) decreases by up to an order of magnitude the SB of the O VIII line in regions populated by non-WHIM, but does not significantly alter the line emission properties in regions of lower overdensity where the WHIM typically resides. Reducing the artificially large brightness of large density regions would decrease the noise level and increase the probability of detecting the emission line systems associated to the WHIM.

From these considerations, we conclude that our WHIM models are adequate to assess the detectability through O VII/O VIII line pairs of the WHIM in emission and are confident that theoretical uncertainties are effectively bracketed, perhaps too generously, in the scatter among B1 and B2 model predictions. On the other hand, the gas distribution in the phase-space (Figure 4) and in the $3 \mathrm{D}$ space (Figure 7 and 8 ), especially in regions of high density and moderate temperature, might not be accurate because of the approximate treatment of gas cooling in the simulations.

The results presented in this paper assume collisional ionization equilibrium. This is a reasonable hypothesis since large part of $\mathrm{O}$ VII and O VIII lines that nextgeneration instruments are expected to detect are likely to be produced by warm-hot gas in high density environments where the CIE is a fairly good assumption $\left(\delta_{g} \gtrsim 100\right.$ and $\left.T \gtrsim 2 \times 10^{6} \mathrm{~K}\right)$. The validity of the CIE hypothesis can be directly checked by analyzing a number of spectral features. One is the relative comparison of the intensity of the resonant, forbidden and intercombination lines of the O VII triplet, which also provide an estimate of the gas temperature, alternative to that obtained from the O VIII/O VII ratio (see, e.g., Porquet et al. 2001). The possibility of performing this test, however, is limited to the few cases in which the components of the triplets can be unambiguously detected. ¿From the analysis of the mock spectra, only in $30 \%$ of the cases can we detect both the resonant and the forbidden lines. The weaker intercombination lines 
is only detected for $\sim 10 \%$. If photo-ionization plays a role, O VIII line is also emitted from lower temperature gas, in addition to O VII triplets. Therefore, the number of detected line systems would increase because lower density $(\delta \lesssim 200)$ regions becomes also detectable. The ionization state of the gas in this low density regions would be affected and the temperature would be overestimated under the assumption of CIE.

Our analysis of the spatial distribution of the line emission systems presented in $\S[5$ is rather qualitative. A more quantitative analysis, based on the mock spectra described in this work, is presented in Ursino et al. (2010a) in which it is shown that the number density of detectable emission line systems is large enough to characterize the distribution by means of the spatial twopoint correlation function. It is also shown that the two-point correlation function of the WHIM is significantly different from that of the non-WHIM and similar to that of galaxies, to which the line-emitting WHIM is associated. Its correlation properties do not seem to evolve significantly in the redshift range $z=[0,0.5]$ and the dynamical state, induced from the velocity-driven anisotropies in the observed clustering, is consistent with that of a gas coherently falling toward larger structures.

The main results of this work are summarized as follows. While the analyses are based on the specification of CRIS onboard EDGE or Xenia, essentially the same conclusions are applicable for DIOS or ORIGIN, which are equipped with a spectrometer of slightly different FOV, angular resolution, and effective area.

- The WHIM can be detected in emission through O VII and O VIII lines. Unambiguous detection of the line systems requires the simultaneous detection of both lines, each one with statistical significance above $5 \sigma$. With a $1 \mathrm{Ms}$ observation and in a FOV of $1 \mathrm{deg}^{2}$ we expect to detect $\sim 640 \mathrm{O} \mathrm{VII} \mathrm{+}$ $\mathrm{O}$ VIII line emitting systems. A significant number of detections $\left(\sim 300\right.$ per $\left.\mathrm{deg}^{2}\right)$ is still expected with a much shorter $(100 \mathrm{ks})$ observation. These results are in broad agreement with those of Cen \& Fang (2006).

- A significant number of these line systems, however, trace gas which is hotter or denser than the WHIM $(\delta>1000)$. The number of line systems contributed by the WHIM is $\sim 65 \%$ (i.e., $\sim 430$ $\mathrm{deg}^{-2}$ ) for a $1 \mathrm{Ms}$ observation. The fraction drops to $\sim 20 \%$ when the exposure time is reduced to $100 \mathrm{ks}$. In other words, deep observations are required to detect a number of line systems large enough to investigate the statistical properties of the WHIM. Shorter observations would preferentially probe hotter gas in high density environments associated to clusters or groups.

- The expected large number of detections is robust. If one considers the very conservative model B1, which we have implemented to assess theoretical uncertainties, the number of line systems above $5 \sigma$ detection threshold is still large $(\sim 480$ $\mathrm{deg}^{-2}$ ), $40 \%$ of which contributed by the WHIM. The difference between model B2 and model B1 provides generous estimate of the theoretical uncer- tainties, significantly larger than the scatter among the models predictions of Cen \& Fang (2006).

- The number of detectable systems is affected by contamination from spurious lines and by the level of the continuum that may vary with energy and direction. It is confirmed that a more rigorous detection procedure that accounts for both effects misses only $\sim 30 \%$ of the line systems that are originally selected.

- The number of detectable emission lines decreases with the energy resolution. However, even with an energy resolution of $7 \mathrm{eV}$, already achieved by the microcalorimeter on board Suzaku, one expects to detect $\sim 160 \quad \mathrm{O}$ VII+O VIII line systems per $\mathrm{deg}^{2}$ in $1 \mathrm{Ms}$ observation.

- Detectable line systems preferentially trace the denser regions of the WHIM with typical gas overdensity of $\delta_{g} \sim 100$, typically associated to virialized structures like galaxies, groups, and the outskirts of galaxy clusters. Increasing the instrumental sensitivity will not change these results significantly since the bulk of the WHIM that resides in lower density environment is characterized by a very low line emissivity. These results agree with those of Bertone et al. (2010) and illustrates the importance of studying the WHIM both in emission (to assess its spatial distribution and thermal state) and in absorption (to probe low density regions and close the baryon budget).

- In the region where CIE holds, the temperature of the gas associated to the detectable emission systems can be reliably estimated from the $\mathrm{O}$ VII/O VIII line ratio in the range of (1$5) \times 10^{6} \mathrm{~K}$, with typical random errors of $\sim 20$ $\%$. The measured temperature trace the O VIIemission weighted temperature of the gas, which, for $T=(1-3) \times 10^{6} \mathrm{~K}$, probes the bulk of the gas in the emitting region.

- With 400-600 line emission systems detected per square degree, one can trace the spatial distribution of the line emitting gas, a large fraction of which is in the warm-hot phase. The visual inspection of the emission maps confirms that the selected line systems trace the large scale filamentary structure of the gas with typical overdensity $\rho_{\text {gas }} /\left\langle\rho_{\text {gas }}\right\rangle \geq 75$. Since line emission is associated to the denser part of the WHIM, the tracing is rather sparse and concentrated around the virialized structures that punctuate the filaments. Moreover, because of the unfavorable ionization balance, the tracing is biased against the largest virialized structures such as galaxy clusters that are typically found at the nodes of the network of filaments.

- The observed $d N / d z$ relation can be compared to theoretical predictions to discriminate among WHIM models and constrain the physical processes that regulate its thermal state and chemical composition. As an example we have shown that the $d N / d z$ statistics is capable of discriminating among 
different metallicity models with a significance of $3-5 \sigma$ with a $1 \operatorname{deg}^{2}$ observation, depending on the energy resolution. Further constraints will be provided by the analysis of the $3 \mathrm{D}$ distribution of the line emission systems and by combining these results with those obtained from the analysis of the absorption spectra.

The authors would like to thank an anonymous referee for the important comments that helped improving the manuscript. The authors are grateful to Stefano Borgani for providing us the outputs of Borgani et al. (2004) hydrodynamical simulation realized using the IBM-SP4 machine at the "Consorzio Interuniversitario del NordEst per il Calcolo Elettronico" (CINECA), with CPU time assigned thanks to an INAF-CINECA grant. YT appreciates the fruitful discussion with Hiroshi Yoshitake on the SWCX. EU acknowledges financial contribution from contracts ASI-INAF I/088/06/0 WP 15300 and ASI-INAF I/088/06/0 TH-018. MV is supported by a PRIN-INAF, a PRIN-MIUR, INFN-PD51 and, the ERC-StG "cosmoIGM".

SRON is supported financially by NWO, the Netherlands Organization for Scientific Research. This work is also supported by JSPS grant-in-aid (KAKENHI 20840051, 22111513).

\section{REFERENCES}

Anders, E., \& Grevesse, N. 1989, Geochim. Cosmochim. Acta, 53, 197

Barlow, T. A., \& Tytler, D. 1998, AJ, 115, 1725

Bertone, S., Schaye, J., Dalla Vecchia, C., Booth, C. M., Theuns, T., \& Wiersma, R. P. C. 2010, MNRAS, 407, 544

Bodewits, D., Christian, D. J., Torney, M., Dryer, M., Lisse, C. M., Dennerl, K., Zurbuchen, T. H., Wolk, S. J., Tielens, A. G. G. M., \& Hoekstra, R. 2007, A\&A, 469, 1183

Borgani, S., Murante, G., Springel, V., Diaferio, A., Dolag, K., Moscardini, L., Tormen, G., Tornatore, L., \& Tozzi, P. 2004, MNRAS, 348, 1078

Branchini, E., Ursino, E., Corsi, A., Martizzi, D., Amati, L., den Herder, J. W., Galeazzi, M., Gendre, B., Kaastra, J. Moscardini, L., Nicastro, F., Ohashi, T., Paerels, F., Piro, L., Roncarelli, M., Takei, Y., \& Viel, M. 2009, ApJ, 697, 328 Bregman, J. N. 2007, ARA\&A, 45, 221

Buote, D. A., Zappacosta, L., Fang, T., Humphrey, P. J., Gastaldello, F., \& Tagliaferri, G. 2009, ApJ, 695, 1351

Burles, S., \& Tytler, D. 1997, AJ, 114, 1330

Burrows, D. N., Hartmann, D., Kouvelioutou, C., Piro, L., den Herder, J., \& Ohashi, T. 2010, in Society of Photo-Optical Instrumentation Engineers (SPIE) Conference Series, Vol. 7732, Society of Photo-Optical Instrumentation Engineers (SPIE) Conference Series

Cen, R., \& Fang, T. 2006, ApJ, 650, 573

Cen, R., \& Ostriker, J. P. 1999a, ApJ, 519, L109

-. 1999b, ApJ, 514, 1

-. 2006, ApJ, 650, 560

Chen, X., Weinberg, D. H., Katz, N., \& Davé, R. 2003, ApJ, 594, 42

Croft, R. A. C., Di Matteo, T., Davé, R., Hernquist, L., Katz, N., Fardal, M. A., \& Weinberg, D. H. 2001, ApJ, 557, 67

Danforth, C. W. 2009, in American Institute of Physics Conference Series, Vol. 1135, American Institute of Physics Conference Series, ed. M. E. van Steenberg, G. Sonneborn, H. W. Moos, \& W. P. Blair , 8-15

Danforth, C. W., \& Shull, J. M. 2005, ApJ, 624, 555

-. 2008, ApJ, 679, 194

Danforth, C. W., Stocke, J. T., \& Shull, J. M. 2010, ApJ, 710, 613

Davé, R., Cen, R., Ostriker, J. P., Bryan, G. L., Hernquist, L., Katz, N., Weinberg, D. H., Norman, M. L., \& O'Shea, B. 2001, ApJ, 552, 473

Fang, T., Buote, D. A., Humphrey, P. J., Canizares, C. R., Zappacosta, L., Maiolino, R., Tagliaferri, G., \& Gastaldello, F. 2010, ApJ, 714, 1715

Fang, T., Davis, D. S., Lee, J. C., Marshall, H. L., Bryan, G. L., \& Canizares, C. R. 2002, ApJ, 565, 86

Fujimoto, R., Mitsuda, K., Mccammon, D., Takei, Y., Bauer, M., Ishisaki, Y., Porter, S. F., Yamaguchi, H., Hayashida, K., \& Yamasaki, N. Y. 2007, PASJ, 59, 133

Fujimoto, R., Takei, Y., Tamura, T., Mitsuda, K., Yamasaki, N. Y., Shibata, R., Ohashi, T., Ota, N., Audley, M. D., Kelley, R. L., \& Kilbourne, C. A. 2004, PASJ, 56, L29

Fukugita, M., Hogan, C. J., \& Peebles, P. J. E. 1998, ApJ, 503, 518

Fukugita, M., \& Peebles, P. J. E. 2004, ApJ, 616, 643
Galeazzi, M., Gupta, A., Covey, K., \& Ursino, E. 2007, ApJ, 658, 1081

Galeazzi, M., Gupta, A., \& Ursino, E. 2009, ApJ, 695, 1127

Gehrels, N. 1986, ApJ, 303, 336

Gupta, A., Galeazzi, M., Koutroumpa, D., Smith, R. K., \& Lallement, R. 2009, ApJ, 707, 644

Haardt, F., \& Madau, P. 1996, ApJ, 461, 20

Henley, D. B., \& Shelton, R. L. 2008, ApJ, 676, 335

Henley, D. B., Shelton, R. L., \& Kuntz, K. D. 2007, ApJ, 661, 304

Hickox, R. C., \& Markevitch, M. 2007a, ApJ, 671, 1523

- 2007b, ApJ, 661, L117

Kaastra, J. S., Werner, N., Herder, J. W. A. d., Paerels, F. B. S., de Plaa, J., Rasmussen, A. P., \& de Vries, C. P. 2006, ApJ, 652,189

Kawahara, H., Yoshikawa, K., Sasaki, S., Suto, Y., Kawai, N., Mitsuda, K., Ohashi, T., \& Yamasaki, N. Y. 2006, PASJ, 58, 657

Kelley, R. L., Mitsuda, K., Allen, C. A., Arsenovic, P., Audley, M. D., Bialas, T. G., Boyce, K. R., Boyle, R. F., Breon, S. R., Brown, G. V., Cottam, J., Dipirro, M. J., Fujimoto, R., Furusho, T., Gendreau, K. C., Gochar, G. G., Gonzalez, O., Hirabayashi, M., Holt, S. S., Inoue, H., Ishida, M., Ishisaki, Y., Jones, C. S., Keski-Kuha, R., Kilbourne, C. A., McCammon, D., Morita, U., Moseley, S. H., Mott, B., Narasaki, K., Ogawara, Y., Ohashi, T., Ota, N., Panek, J. S., Porter, F. S., Serlemitsos, A., Shirron, P. J., Sneiderman, G. A., Szymkowiak, A. E., Takei, Y., Tveekrem, J. L., Volz, S. M., Yamamoto, M., \& Yamasaki, N. Y. 2007, PASJ, 59, 77

Klypin, A., Hoffman, Y., Kravtsov, A. V., \& Gottlöber, S. 2003, ApJ, 596, 19

Komatsu, E., Dunkley, J., Nolta, M. R., Bennett, C. L., Gold, B., Hinshaw, G., Jarosik, N., Larson, D., Limon, M., Page, L., Spergel, D. N., Halpern, M., Hill, R. S., Kogut, A., Meyer, S. S., Tucker, G. S., Weiland, J. L., Wollack, E., \& Wright, E. L. 2009, ApJS, 180, 330

Koutroumpa, D., Lallement, R., Kharchenko, V., Dalgarno, A., Pepino, R., Izmodenov, V., \& Quémerais, E. 2006, A\&A, 460, 289

Kravtsov, A. V., Klypin, A., \& Hoffman, Y. 2002, ApJ, 571, 563

Mannucci, F., Bonnoli, G., Zappacosta, L., Maiolino, R., \& Pedani, M. 2007, A\&A, 468, 807

Mathur, S., Weinberg, D. H., \& Chen, X. 2003, ApJ, 582, 82

McCammon, D., Almy, R., Apodaca, E., Bergmann Tiest, W., Cui, W., Deiker, S., Galeazzi, M., Juda, M., Lesser, A., Mihara, T., Morgenthaler, J. P., Sanders, W. T., Zhang, J., Figueroa-Feliciano, E., Kelley, R. L., Moseley, S. H. Mushotzky, R. F., Porter, F. S., Stahle, C. K., \& Szymkowiak, A. E. 2002, ApJ, 576, 188

Morrison, R., \& McCammon, D. 1983, ApJ, 270, 119

Narayanan, A., Savage, B. D., \& Wakker, B. P. 2010a, ApJ, 712, 1443

Narayanan, A., Wakker, B. P., Savage, B. D., Keeney, B. A., Shull, J. M., Stocke, J. T., \& Sembach, K. R. 2010b, ApJ, 721, 960

Nicastro, F., Mathur, S., Elvis, M., Drake, J., Fang, T., Fruscione, A., Krongold, Y., Marshall, H., Williams, R., \& Zezas, A. 2005, Nature, 433, 495 
Ohashi, T., Ishisaki, Y., Ezoe, Y., Sasaki, S., Kawahara, H., Mitsuda, K., Yamasaki, N. Y., Takei, Y., Ishida, M., Tawara, Y., Sakurai, I., Furuzawa, A., Suto, Y., Yoshikawa, K., Kawai, N., Fujimoto, R., Tsuru, T. G., Matsushita, K., \& Kitayama, T. 2010, in Society of Photo-Optical Instrumentation Engineers (SPIE) Conference Series, Vol. 7732, Society of Photo-Optical Instrumentation Engineers (SPIE) Conference Series

Oppenheimer, B. D., \& Davé, R. 2008, MNRAS, 387, 577

Paerels, F., Kaastra, J., Ohashi, T., Richter, P., Bykov, A., \& Nevalainen, J. 2008, Space Science Reviews, 134, 405

Piro, L., den Herder, J. W., Ohashi, T., Amati, L., Atteia, J. L., Barthelmy, S., Barbera, M., Barret, D., Basso, S., Boer, M., Borgani, S., Boyarskiy, O., Branchini, E.,

Branduardi-Raymont, G., Briggs, M., Brunetti, G.

Budtz-Jorgensen, C., Burrows, D., Campana, S., Caroli, E., Chincarini, G., Christensen, F., Cocchi, M., Comastri, A., Corsi, A., Cotroneo, V., Conconi, P., Colasanti, L., Cusumano, G., de Rosa, A., Del Santo, M., Ettori, S., Ezoe, Y., Ferrari, L., Feroci, M., Finger, M., Fishman, G., Fujimoto, R., Galeazzi,

M., Galli, A., Gatti, F., Gehrels, N., Gendre, B., Ghirlanda, G., Ghisellini, G., Giommi, P., Girardi, M., Guzzo, L., Haardt, F., Hepburn, I., Hermsen, W., Hoevers, H., Holland, A., in't Zand, J., Ishisaki, Y., Kawahara, H., Kawai, N., Kaastra, J., Kippen, M., de Korte, P. A. J., Kouveliotou, C., Kusenko, A., Labanti, C., Lieu, R., Macculi, C., Makishima, K., Matt, G., Mazzotta, P., McCammon, D., Méndez, M., Mineo, T., Mitchell, S., Mitsuda, K., Molendi, S., Moscardini, L., Mushotzky, R., Natalucci, L., Nicastro, F., O'Brien, P., Osborne, J., Paerels, F., Page, M., Paltani, S., Pareschi, G., Perinati, E., Perola, C., Ponman, T., Rasmussen, A., Roncarelli, M., Rosati, P. Ruchayskiy, O., Quadrini, E., Sakurai, I., Salvaterra, R., Sasaki, S., Sato, G., Schaye, J., Schmitt, J., Sciortino, S., Shaposhnikov, M., Shinozaki, K., Spiga, D., Suto, Y., Tagliaferri, G., Takahashi, T., Takei, Y., Tawara, Y., Tozzi, P., Tsunemi, H., Tsuru, T., Ubertini, P., Ursino, E., Viel, M., Vink, J., White, N., Willingale, R., Wijers, R., Yoshikawa, K., \& Yamasaki, N. 2009, Experimental Astronomy, 23, 67

Porquet, D., Mewe, R., Dubau, J., Raassen, A. J. J., \& Kaastra, J. S. 2001, A\&A, 376, 1113

Rasmussen, A. P., Kahn, S. M., Paerels, F., Herder, J. W. d., Kaastra, J., \& de Vries, C. 2007, ApJ, 656, 129

Rauch, M. 1998, ARA\&A, 36, 267

Roncarelli, M., Moscardini, L., Tozzi, P., Borgani, S., Cheng, L. M., Diaferio, A., Dolag, K., \& Murante, G. 2006, MNRAS, 368,74

Schaye, J., Dalla Vecchia, C., Booth, C. M., Wiersma, R. P. C.,

Theuns, T., Haas, M. R., Bertone, S., Duffy, A. R., McCarthy, I. G., \& van de Voort, F. 2010, MNRAS, 402, 1536

Shen, S., Wadsley, J., \& Stinson, G. 2010, MNRAS, 407, 1581
Smith, R. K., Bautz, M. W., Edgar, R. J., Fujimoto, R., Hamaguchi, K., Hughes, J. P., Ishida, M., Kelley, R., Kilbourne, C. A., Kuntz, K. D., McCammon, D., Miller, E., Mitsuda, K., Mukai, K., Plucinsky, P. P., Porter, F. S., Snowden, S. L., Takei, Y., Terada, Y., Tsuboi, Y., \& Yamasaki, N. Y. 2007, PASJ, 59, S141

Smith, R. K., Brickhouse, N. S., Liedahl, D. A., \& Raymond, J. C. 2001, ApJ, 556, L91

Smith, R. K., Edgar, R. J., Plucinsky, P. P., Wargelin, B. J., Freeman, P. E., \& Biller, B. A. 2005, ApJ, 623, 225

Spergel, D. N., Verde, L., Peiris, H. V., Komatsu, E., Nolta, M. R., Bennett, C. L., Halpern, M., Hinshaw, G., Jarosik, N., Kogut, A., Limon, M., Meyer, S. S., Page, L., Tucker, G. S., Weiland, J. L., Wollack, E., \& Wright, E. L. 2003, ApJS, 148, 175

Springel, V. 2005, MNRAS, 364, 1105

Springel, V., \& Hernquist, L. 2003, MNRAS, 339, 289

Takei, Y., Henry, J. P., Finoguenov, A., Mitsuda, K., Tamura, T., Fujimoto, R., \& Briel, U. G. 2007, ApJ, 655, 831

Tornatore, L., Borgani, S., Viel, M., \& Springel, V. 2010, MNRAS, 402, 1911

Tripp, T. M., Bowen, D. V., Sembach, K. R., Jenkins, E. B., Savage, B. D., \& Richter, P. 2006, in Astronomical Society of the Pacific Conference Series, Vol. 348, Astrophysics in the Far Ultraviolet: Five Years of Discovery with FUSE, ed. G. Sonneborn, H. W. Moos, \& B.-G. Andersson, 341-+

Tripp, T. M., Sembach, K. R., Bowen, D. V., Savage, B. D., Jenkins, E. B., Lehner, N., \& Richter, P. 2008, ApJS, 177, 39

Ursino, E., Branchini, E., Galeazzi, M., Marulli, F., Moscardini, L., Piro, L., Roncarelli, M., \& Takei, Y. 2010a, MNRAS, submitted, arXiv:1009.5519

Ursino, E., \& Galeazzi, M. 2006, ApJ, 652, 1085

Ursino, E., Galeazzi, M., \& Roncarelli, M. 2010b, ApJ, 721, 46

Viel, M., Branchini, E., Cen, R., Matarrese, S., Mazzotta, P., \& Ostriker, J. P. 2003, MNRAS, 341, 792

Viel, M., Branchini, E., Cen, R., Ostriker, J. P., Matarrese, S., Mazzotta, P., \& Tully, B. 2005, MNRAS, 360, 1110

Vikhlinin, A., \& Forman, W. 1995, ApJ, 455, L109+

Weinberg, D. H., Miralda-Escude, J., Hernquist, L., \& Katz, N. 1997, ApJ, 490, 564

Werner, N., Finoguenov, A., Kaastra, J. S., Simionescu, A., Dietrich, J. P., Vink, J., \& Böhringer, H. 2008, A\&A, 482, L29

Yoshikawa, K., Dolag, K., Suto, Y., Sasaki, S., Yamasaki, N. Y., Ohashi, T., Mitsuda, K., Tawara, Y., Fujimoto, R., Furusho, T., Furuzawa, A., Ishida, M., Ishisaki, Y., \& Takei, Y. 2004, PASJ, 56, 939

Yoshikawa, K., Yamasaki, N. Y., Suto, Y., Ohashi, T., Mitsuda, K., Tawara, Y., \& Furuzawa, A. 2003, PASJ, 55, 879

Yoshino, T., Mitsuda, K., Yamasaki, N. Y., Takei, Y., Hagihara, T., Masui, K., Bauer, M., McCammon, D., Fujimoto, R., Wang, Q. D., \& Yao, Y. 2009, PASJ, 61, 805

Zappacosta, L., Maiolino, R., Mannucci, F., Gilli, R., \& Schuecker, P. 2005, MNRAS, 357, 929

Zappacosta, L., Nicastro, F., Maiolino, R., Tagliaferri, G., Buote, D. A., Fang, T., Humphrey, P. J., \& Gastaldello, F. 2010, ApJ, 717,74 